\title{
LA EMPLEABILIDAD A DEBATE: ¿QUÉ SABEMOS SOBRE LA EMPLEABILIDAD COMO ESTRATEGIA DE CAMBIO SOCIAL?
}

\author{
Lucía Llinares Insa \\ Departamento de Psicología Social, Facultat de Psicologia \\ Universitat de València
}

\section{Ana Isabel Córdoba Iñesta}

Departamento de Psicología Evolutiva y de la Educación, Facultat de Psicologia

Universitat de València

\section{Pilar González-Navarro}

IDOCAL, Universitat de València

\section{RESUMEN}

Los cambios en mundo laboral derivados de la globalización y el neoliberalismo han puesto a la empleabilidad en el centro de atención del desarrollo económico y social. Sin embargo, la falta de consenso en las teorías y las dimensiones que la definen reclaman un análisis de la investigación que permita su uso como estrategia de cambio social. El objetivo de este trabajo es realizar una revisión sistemática de los marcos teóricos sobre empleabilidad. Se ha realizado un análisis de 239 artículos científicos atendiendo a dos áreas temáticas: la historia del concepto y las perspectivas teóricas. Los resultados de esta revisión han permitido sistematizar y comprender las orientaciones teóricas desde las que se ha analizado la empleabilidad, las premisas que toman las diferentes teorías como punto de partida y los indicadores propuestos. A partir de todo ello, se han abierto algunas cuestiones para la reflexión de los agentes sociales vinculados a la educación, la acción social y al empleo.

PALABRAS CLAVE: Empleabilidad, vulnerabilidad, exclusión social, inserción laboral, mercado laboral, desigualdad laboral, cambio social.

CLAVES ECONLIT: H53, L31, Z13.

Cómo citar este artículo/How to cite this article: LLINARES INSA, L., CÓRDOBA IÑESTA, A.I. \& GONZÁLEZ NAVARRO, P.: “La empleabilidad a debate: ¿qué sabemos sobre la empleabilidad como estrategia de cambio social?", CIRIEC-España, Revista Jurídica de Economía Social y Cooperativa, $\mathrm{n}^{0} 36,2020$, pp. 313-363. DOI: 10.7203/CIRIEC-JUR.36.17017. 


\section{DEBATE ON EMPLOYABILITY: WHAT DO WE KNOW ABOUT EMPLOYABILITY AS STRATEGY OF SOCIAL CHANGE?}

\section{EXPANDED ABSTRACT}

Many studies have analyzed the concept of employability. However, its importance and systematization is relatively recent. Firstly, late changes in the economic and labor areas made employability an important subject of study (Gracier, 2001; McQuaid \& Lindsay, 2005; Rentería \& Malvezzi., 2008; Tijssen, Van der Heijden \& Roco, 2008). Secondly, many authors analyze the dimensions and indicators of employability to evaluate and categorize people (for example, Fugate, Kinicki, and Ashforh, 2004; Tijssen, Van der Heijden, and Roco, 2008). Third, the term changes from the focus on the unemployed to the focus on the general population. Fourth, employability has been linked to the acquisition of formal titles from the Government. Fifth, employability became popular in few years.

We don't know any studies that analyze its meaning in the social discourse, focused on the individual responsibility over his employability instead of social agents (Salognon, 2007). To clarify this, an explanatory model of employability emerged to consider employability as a socially constructed historical category and, thus, conditioned by socio-historical and institutional factors: the Bioecological Model of Employability proposed by Llinares-Insa, Córdoba, and Zacarés (2016). This model emphasizes the importance of the socio-structural elements in the genesis of the process. But it goes one step further and focuses on individual-society interaction.

The objective of this study is to define the concept of employability as a social construction from a broad perspective that includes all its repercussions. Thus, we carried out a bibliographic analysis on the scientific approach to the term and the theoretical frameworks, the evolution of the meaning of employability, the context in which it is framed and the contributions of the different approaches. Finally, we present an attempt to the concept, emphasizing its relationship with socio-labor inclusion/exclusion and the labor and social differences of different individuals and/or groups.

We performed a bibliographic search based on the recommendations of the systematic reviews and meta-analyzes of the PRISMA statement (Urrútia and Bonfill, 2010), from 2011 to the present. We reviewed SCOPUS, Proquest Central, DIALNET, PsycARTICLES and Web of Science with the terms 'employabiliy', 'concept' and 'framework' in the title and summary. We included only articles from scientific journals. We excluded papers referred to the measure of employability, questionnaires of employability, or those that analyze employability in a 
specific context or from an international organization or intervention program. We analyzed the content and extracted the most relevant data and made comparative tables according to the similarities and differences.

We organized this review through three big sections focused on well-differentiated characteristics to explain employability:

\section{Historical evolution of the concept}

The concept of employability has recently been integrated into the language, although it emerged decades ago (Serrano, 2000). According to Formichella and London (2005) or Van der Heijde and Van der Heijden (2006) the first publications are from the 1950s and referred to concerns about the position of homeless people in the labor market, such as disabled people. They presented employability as the potential a person has and that allows him to access a job and remain in it.

In the 1960s, Gazier $(1998,2001)$ distinguished three types of employability: a) Sociomedical referred to the distance between the real skills for the employment of people with social, physical or mental disadvantages and the requirements of employment. From this moment, people with some type of deficiency begin to be considered employable. b) The employability of labor policy, developed as an attempt to extend the socio-medical perspective to other disadvantaged groups. c) Fluid employability focused on demand and accessibility to employment in local and national economies; it defined employability as the high probability to find a job (Gazier, 2001).

In the last decades of the 20th century, the employment-based system changed to the worker-centered system (Van der Heijde and Van der Heijden, 2006). In the 1970s, employability focused on the measurement of both professional qualification and mobility deficiencies, measuring the distance between individual characteristics and requirements in the labor market, which promoted employment-oriented intervention programs (Renteria and Malvezzi, 2008).

In the 1980 s and 1990 s the concept of employability focused on the social inclusion of disadvantaged groups and was a reaction to the consequences of high levels of long-term unemployed and a trend towards new types of relationships between employers and employees (McQuaid and Lindsay, 2005).

In the late 1980s, the concept of initiative of employability reflected an acceptance that developing a successful career required the development of transferable skills and the flexibility 
to perform different roles on the job. It focused on the individual and his responsibility to develop those skills and be employable.

Towards the end of the 20th century, there was a leap to the new psychological contract and employability is considered a crucial component to get and maintain employment (Forrier and Sels, 2003). Moreover, international organizations also highlight the importance of employability for reducing social exclusion.

\section{Epistemological perspectives on employability}

We can distinguish two major theoretical perspectives in the analysis of the concept of employability:

A) The individualistic perspective focuses on the individual as responsible for developing their career (Kovalenko et al., 2016). Therefore, its role is to get qualities to be more employable (Fugate and Knicki, 2008). In return, they have the right to demand training and learning opportunities from the company (Montañana, 2005). This one-dimensional perspective labels the worker as employable or non-employable based on her ability to get a job (Finn, 2000). It focuses on the need for employees to adapt to new labor market situations and new types of organization (Formichella and London, 2005; Fugate et al., 2004).

B) The socio-critical perspective presents a critical discourse towards the apparent rationality of the market (Brunet and Pastor, 2003). It claims that employability is a social construction (Castillo, 1996) that begins with and maintains class differences (Brunet and Pastor, 2003; Reid, 2015). According to this perspective, occupational policies are considered ineffective. Public action deregulates the labor market and this maintains and generates new inequalities because the success or failure of their work career is attributed to the individual without taking into account the skills they have and the skills the jobs demand.

C) The bioecological model of employability: an integrative proposal from psychology. Finally, we present a proposal about employability as a process that connects the responsibility of society, companies and the context, the bioecological model on employability as an integrative proposal (Llinares-Insa, Córdoba and Zacarés, 2016). It considers employability as a social construction resulting from the reciprocal interaction between an active and developing biopsychological human organism and the people, objects and symbols of its external environment related to the acquisition and maintenance of employment.

Employability must be understood as a process linked to the person with his/her individual and social history. The model focus on (Bronfenbrenner and Morris, 1998): a) Individual dis- 
positions; b) The resources and positive and negative biopsychological elements that affect the subject's ability to effectively engage in proximal processes; and c) The characteristics of the person that affect the way in which others react to it.

This model emphasizes the importance of the environment; therefore, in disadvantaged and disorganized environments, the impact of proximal processes will be greater on those results that reflect an evolutionary dysfunction (Bronfenbrenner and Morris, 1998). Thus, the development of the person in advantageous and stable environments that transmit contents in accordance with the requirements and parameters imposed by the economic, political, socio-cultural and technological structure are more likely to increase the functional competence of the person and, therefore, employability.

Therefore, the bioecological model achieves the complexity of all types of groups and situations where the term is used, from a young university student to an adult in a work integration social enterprise.

The belief is that there is a standard working person and that everyone can reach it, but this is not real (Clark and Patrickson, 2008). The individual who feels unable to control the situation and who has internalized the institutional discourse may attribute their difficulties of labor integration to their own personal characteristics. This leads to the belief that unemployment is due to their way of being and proceeding, which generates a low level of self-concept, self-esteem and aspirations. As a result, the person can belief the impossibility of playing a valuable role, and this may promote a series of deficiencies in the cognitive, attitudinal and motivational personal areas. For all these reasons, we can affirm that the meaning of the concept of employability is not neutral in any context and that its use requires prior analysis of the symbolic element it evokes.

KEYWORDS: Employability, vulnerability, social exclusion, labour insertion, labour market, social inequalities, social change. 
(pp. 313-363)

\section{SUMARIO $^{1}$}

1. Introducción. 2. Método. 3. Resultados. 3.1. Evolución histórica del término. 3.2. Las corrientes epistemológicas sobre la empleabilidad. 4. Discusión y conclusiones. Referencias bibliográficas.

\section{Introducción}

La empleabilidad y el ser empleable son tópicos que han recibido gran atención en la literatura científica, los discursos políticos y la acción social (Álvarez-González, López Miguens y Caballero, 2017). El neoliberalismo, la globalización y la crisis están generando grandes cambios en el mundo laboral, como es la liberalización de los mercados, la regulación por el laissez faire, la falta de seguridad en el empleo, la disminución de derechos laborales, etc. (Bernad, Martínez y Molpeceres, 2011; Suarez, 2016). Esta situación pone en primera línea el interés por el individuo y el desarrollo de su carrera profesional (Inkson, Gunz, Ganesh \& Roper, 2012; Kendall y French, 2018; Olssen y Peters, 2005). Además, los nuevos modelos de carreras laborales (Davis, Baty, Hippman, Trepanier y Erby, 2019; Mäkikangas, De Cuyper, Mauno y Kinnunen, 2013; Muñiz, 2012) se fundamentan en un discurso en el que la empleabilidad es un elemento central y los cambios en el plano económico y laboral han propiciado que la empleabilidad sea un tema importante de estudio (ver, por ejemplo, Gracier, 2001; McQuaid y Lindsay, 2005; Midtsundstad, 2019; Rentería y Malvezzi, 2008; Tijssen, Van der Heijden y Roco, 2008). Así, la empleabilidad es presentada como una alternativa a la seguridad en el empleo y la reducción del desempleo (Bonfiglioli, Moir y Ambrosini, 2006; Bernstrom, Drange \& Mamelund, 2019).

La empleabilidad y su conceptualización ha sido ampliamente estudiadas y son muchas las áreas de conocimiento que la analizan (Peiró y Yeves, 2017). Además, las teorías sobre empleabilidad han ido aumentando en complejidad y multidimensionalidad y sus definiciones han ido incorporando componentes nuevos en función del colectivo analizado (Williams, Dodd, Steele y Randall, 2016). Esto ha generado un panorama de gran confusión y ambigüedad teórica, así como, poca integración en sus contenidos. El resultado ha sido una falta de acuerdo en el constructo y una gran cantidad de usos (De Lara y Andrade, 2008; Hillage y Pollard, 1998; McQuaid

1. Este trabajo se deriva del proyecto que lleva por nombre 'EMPLEAM: Evalúa y mejora la empleabildiad' concedido en la 'Convocatòria d'Ajudes per a la Realització de Projectes de Prova de Concepte 209. Programa Valoritza i Transfereix’ del Vicerectorat d'Innovació i Transferència de la Universitat de València. 
y Lindsay, 2005; Moreland, 2006). Todo ello, reclaman un análisis que organice este panorama tan difuso.

Existen algunas revisiones sobre temas conceptuales y/o empíricos de la empleabilidad. Éstas tratan aspectos concretos como, por ejemplo, la empleabilidad de los graduados (ver p.e. Tomlinson, 2012), en educación superior (ver p.e. Fahimirad, Nair, Kotamjani, Mahdinezhad y Fengf, 2019), autores que enfocan la empleabilidad únicamente a nivel individual (ver p.e. Williams et al., 2016), se centran en las intervenciones para su fomento (ver p.e. Hazelzet, Picco, Houkes, Bosma y de Rijk, 2019), para el aprendizaje adulto (ver p.e. Midtsundstad, 2019), para áreas laborales concretas (ver p.e. Sisodia y Agrawal, 2019). De forma más general, algunas investigaciones delimitan un número de ańos, aunque esto restringe el número de aportaciones presentadas (ver p.e. Artess, Holey y Mellors-Bourne, 2017) y otras se centran en aspectos generales como su definición (Suarez, 2016). Sin embargo, no hemos encontrado ninguna revisión que incluya los marcos teóricos relevantes que permitan definirla y situarla en el contexto de la persona desde una perspectiva holística. Este es el objetivo del trabajo que se presenta, llevar a cabo una revisión sistemática de la literatura de los marcos teóricos sobre empleabilidad.

El análisis científico mayoritario de la empleabilidad la ha presentado como producto de las diferencias individuales. Esto se observa en la evolución histórica de la noción de empleabilidad y su unión con la exclusión social (Gómez-Torres, Rodríguez-Santero y Gil-Flores, 2019). El concepto de empleabilidad se utilizó históricamente para separar los aptos de los ineptos (Rentería y Malvezzi, 2008). En este sentido, se analizan las características individuales y las experiencias que distinguen a las personas empleables de las que no lo son. Se centran en el análisis de sus dimensiones (por ejemplo, Fugate Kinicki y Ashforh, 2004; Tijssen, Van der Heijden y Rocco, 2008) y los indicadores de la empleabilidad que tienen como eje central la evaluación y categorización de las personas (ver, por ejemplo, la Escala de Percepción de la Empleabilidad de Daniels, D’Andrea y Gaughen, 1998; la Escala de Empleabilidad de De Lara y Andrade, 2008; el Instrumento para Medir la Empleabilidad de Van de Heijden y Van der Heijden, 2005; la Escala de Autopercepción de la Empleabilidad de Rothwell y Arnold, 2007). También hay estudios que relacionan la empleabilidad con otras variables psicológicas relevantes de la persona (e.g. Budzynski-Seymour, Conway, Wade, Lucas, Jones, Mann y Steele, 2020), analizan variables mediadoras (e.g. Pitan \& Muller, 2019) y moderadoras de la empleabilidad (e.g. Van der Heijden y Spurk, 2019) y también la analizan como variable moderadora (e.g. Yeves, Bragstede, Cortes, Merino y Cavada, 2019) o mediadora (e.g. Akkermans, Tims, Beijer y De Cuiper, 2019), como motor del rendimiento (e.g. Philippaers, De Cuiper y Forrier, 2019), etc. Otros estudios analizan sus antecedentes (e.g. Kittisak, Thana- 
porn y Parinya, 2019), los servicios especializados que la fomentan (e.g. Faulkner, Begum, Warren, 2019), etc. Así pues, la producción en los últimos años ha sido muy elevada; sin embargo, no todos los estudios presentan el significado que le atribuyen a la empleabilidad y sus indicadores son completamente diferentes. Esto proporciona ambigüedad en su interpretación.

Otra línea de publicaciones sobre empleabilidad es la que se centra en los colectivos que se encuentran afectados (ver, por ejemplo, Clarke y Patrickson, 2008). Las primeras publicaciones sobre el concepto de empleabilidad suponen un acercamiento a la consideración de las personas con posibilidades de ser excluidas del mercado de trabajo (Van der Heijde y Van der Heijden, 2006) y por ello se analizaban los grupos poblacionales potencialmente no empleables (ver, por ejemplo, Aceña y Caparrós, 2018; Formichella y London, 2005; Garzso, 2007).

Actualmente, y debido a las cifras de desempleo y a la emergencia de nuevas formas de empleo y de carrera laboral, este concepto se ha ampliado a toda la población, aunque a los que realmente afecta es a la población vulnerable (Santana, Alonso y Feliciano, 2016). Desde esta perspectiva se analizan, por ejemplo, los referentes sociales y culturales que hacen más probable la vulnerabilidad y se analizan sus implicaciones (ver, por ejemplo, Belt y Richardson, 2005). También hay estudios que analizan la empleabilidad de los estudiantes universitarios (e.g. Koe, 2018), de los graduados (ver, por ejemplo, Belwal, Priyadarshi y Al Fazari, 2016), personas con una discapacidad reconocida (e.g. Ebuenyi, Guxens, Ombati, Bunders-Aelen y Regeer, 2019), refugiados (e.g. Scheibelhofer y Täubig, 2019), mujeres (e.g. Sarmento, Ferreira, Amorim, Madaleno y Pimentel, 2019), migrantes (e.g. Wu, 2019), etc. También los hay que comparan colectivos (e.g. Abdi, Eskandari y Jalali, 2019). En este sentido, la evolución del término pasa de centrarse en personas y/o grupos que se sitúan en el margen del sistema, los desempleados, a extenderse a toda la población. Así pues, es necesaria una sistematización y clarificación de las bases que la explican y una concreción en los diversos estudios sobre qué se está analizando y evaluando.

Esta circunstancia se observa también en la confusión actual entre empleabilidad y títulos formales. La empleabilidad se ha identificado con la adquisición de títulos formales expedidos por los Estados (Peiró y Yeves, 2017). La relación entre la empleabilidad y la educación formal, informal y no formal ha existido siempre hasta el punto de ser considerada la causa primordial en su desarrollo y el instrumento base de su fomento (ver, por ejemplo, Ducci, 1998; Santana et al., 2016). En este sentido, se ha transmitido que las personas con mayor nivel de instrucción tienen rentas más elevadas y pueden desarrollar más sus habilidades. La obtención de títulos formales está en la base de la relación directa con el acceso al empleo. De esta manera, la empleabilidad justifica la distancia social de las personas sin estudios formales. 
Un ejemplo de ello es el modelo de 'hacer fila' de Thurow (1972). En el modelo se propone que existe competencia laboral y que las personas se ordenan en una fila en función de ciertas características (por ejemplo, la educación). Las personas más capacitadas estarían al principio de la fila. En este sentido, la educación es considerada como el garante de la promesa del trabajo bien remunerado y es una competencia a formar (por ejemplo, Rae, 2007; Smith, Mcknigh y Naylor, 2000; Zhiwen y Van der Heijden, 2008). Ante ello, las universidades (e.g. Terzalory y Oyekunle, 2019) con programas concretos (ver, por ejemplo, Clarke, 2017), los curricula (e.g. Bedford \& Bell, 2019) y las actividades académicas (ver por ejemplo, Pitan y Muller, 2019) han sido contextos clave. Desde una perspectiva crítica, sin embargo, se presenta un panorama completamente diferente. Algunos autores ponen de manifiesto que no siempre se corresponde la obtención de títulos formales con un trabajo, ya que el cumplimiento de la promesa del trabajo no está por sí misma garantizada (Brown y Hesketh, 2004). La empleabilidad no es considerada similar al número de títulos formales obtenidos, pero puede ser fomentada. También se ponen de manifiesto los silencios y las ambigüedades de las destrezas que se forman en los curricula para el fomento de la empleabilidad (Butterwick y Benjamin, 2006). Esto reclama la revisión de los marcos teóricos que conceptualizan la empleabilidad porque parece que ambas perspectivas hablan de un mismo tópico con sentido diferente.

La implicación práctica relevante es dotar a toda la población y al contexto de las herramientas apropiadas para el fomento de la empleabilidad en las sociedades para que éstas sean sostenibles. En relación a ello, hay estudios que analizan aspectos tan interesantes como las implicaciones para el cambio en los sistemas formativos (e.g. Espinoza, González, McGinn y Sandoval, 2020), la repercusión de la empleabilidad en el desarrollo sostenible (ver p.e. Rozalía, Blaga y Matis, 2019), en la inclusión laboral (ver p.e. Storme, De Lannoy, Leibbrandt, De Boexk y Mudiriza, 2019), etc. Sin embargo, la mayor parte de ellos no señalan su conceptualización de la empleabilidad y, por tanto, no concretan qué están analizando, ni evaluando. Además, para que este tipo de estudios resultara interesante, la empleabilidad debería ser un constructo que se pueda evaluar de la misma manera en todo tipo de colectivo. Esto permitiría el análisis conjunto de todas las consecuencias psicosociales y educativas en un contexto globalizado y neoliberal. Para ello, se requiere la concreción de la perspectiva teórica desde la que se plantea la evaluación y su fomento en cualquier contexto formativo (universidad, formación profesional, formación permanente, formación de adultos, etc.).

Quizá esta situación se debe a que la empleabilidad es un término que se ha popularizado en pocos años, ha pasado de ser propio de las disciplinas científicas a formar parte de las representaciones sociales de la población. De hecho, es un tópico que surge y se desarrolla para justificar el desempleo, el acceso al empleo y las dife- 
rencias laborales (De Lara y Andrade, 2008). No conocemos estudios que analicen su significado en el discurso social, pero éste apunta a la responsabilidad individual de la persona sobre su trayectoria laboral y quita la responsabilidad al resto de agentes sociales (Salognon, 2007). De este modo, el ser empleable aumenta la probabilidad de éxito del empleo (Berntson, Sverke y Marklund, 2006; Fugate et al., 2004), ya que la empleabilidad lleva a la consecución del empleo (Clarke y Patrickson, 2008). Este discurso hace que la responsabilidad de la falta de empleo y el desempleo sea de la persona y no del resto de los agentes sociales implicados como el Estado o las empresas (ver, por ejemplo Nauta, van Vianen, Van del Heijden, Van Dam y Willemsen, 2009; Salognon, 2007). Posteriormente, algunos artículos comienzan a analizar la responsabilidad de la empresa y de quien emplea (ver, por ejemplo, Attarwala y Balasubramaniam, 2019; Baruch, 2001; Ellig, 1998; Sjollema, 2007; Van Buren, 2003). Desde una perspectiva más social se pone de relieve la importancia de la sociedad en general (ver, por ejemplo, Bollérot, 2001) y del Estado (ver, por ejemplo, Brunet y Pastor, 2003; Butterwick y Benjamin, 2006). Carrieri y Sarsur (2002) plantean que la empleabilidad es un recurso y una estrategia adaptada por la alta administración para transferir la responsabilidad de contratación y despido de la organización a la persona que trabaja. En este sentido, más que una capacidad o cualidad individual, la noción de empleabilidad se convierte en un recurso para la explicación de la inclusión/exclusión laboral (Hirata, 1997). Esta situación requiere un análisis exhaustivo de la comprensión actual de la empleabilidad comenzando por una revisión sobre lo que la literatura científica entiende por empleabilidad y el marco teórico que la explica.

A partir de todo lo expuesto, se constata que la empleabilidad es un tópico que ha generado gran interés en diferentes disciplinas. Sin embargo, pese al volumen de literatura científica que se ha publicado no existe un área de conocimiento concreta y hay pocos estudios que hayan elaborado una integración de sus contenidos (ver, por ejemplo, Llinares, Zacarés y Córdoba, 2016). Suarez (2016), por su parte, realizó una revisión de la literatura desde el año 2000 sobre el concepto de empleabilidad en bases de datos generales. Sin embargo, esto supone una limitación tanto a nivel temporal, porque resulta restrictivo, como a nivel de contenido, ya que no se atiende a las bases de datos de Ciencias Sociales y de Psicología.

Otras revisiones teóricas realizadas atienden a la estructuración de alguna de sus áreas de aplicabilidad como, por ejemplo, los universitarios, pero no al conjunto de los estudios sobre el mismo (ver, por ejemplo, Orellana, 2018). Junto a ello, no todos los estudios que analizan la empleabilidad y/o la evalúan delimitan el concepto ni la perspectiva de la que parten (ver, por ejemplo, Atkins, 1999; Sabin y Hansvick, 1999). Este hecho deriva en la falta de acuerdo en el concepto y el uso del término 
en los estudios científicos. Además, la falta de unanimidad en su conceptualización lleva a dificultades en su delimitación terminológica (Suarez, 2016).

Así pues, el objetivo de este trabajo es realizar una revisión sistemática de los estudios que propongan algún marco teórico sobre empleabilidad, identificando sus premisas y sus indicadores. Esto es relevante a nivel teórico, ya que como señala Kennedy (2007), las revisiones sistemáticas son una herramienta útil para hacer avances en el tópico que se analiza. A nivel práctico también resulta relevante porque permite la práctica social basada en la evidencia y es una herramienta fundamental en la toma de decisiones para todos los agentes sociales.

En este sentido, el fomento de la empleabilidad es un objetivo de las agendas de política social y económica tanto a nivel internacional como nacional. Y este estudio pretende ayudar a las reformas sociales de forma que se puedan ver las barreras de cristal que limitan a los colectivos más vulnerables, pudiendo llegar a ser una herramienta para la inclusión socio-laboral. Resulta, pues, necesaria para todos los profesores universitarios que tienen la obligación de incluirla en sus curricula, agentes sociales que trabajan en la inclusión laboral, estados que pretenden general sociedades sostenibles e igualitarias, etc. Una sociedad que pretenda conseguir los Objetivos de Desarrollo Sostenible del 25 de septiembre de 2015 de la Organización de las Naciones Unidas y la construcción de la Sociedad del Conocimiento de la Declaración de Bolonia en 1999 necesita una forma similar de conocer, comprender, evaluar y fomentar la empleabilidad.

\section{Método}

Este estudio pretende la revisión sistemática de los estudios científicos que proponen un marco teórico sobre empleabilidad. El objetivo es sintetizar la información científica disponible para poder organizarla y plasmarla de forma sistemática. La búsqueda bibliográfica realizada se basa en las recomendaciones de las Revisiones sistemáticas y metaanálisis de la declaración PRISMA (Urrútia y Bonfill, 2010). También se tienen en cuenta las etapas para revisiones sistemáticas y metaanálisis propuestas por Ferreira, Urrútia y Alonso-Coello (2011).

El elemento clave para la formulación de la pregunta de interés de la revisión es el objetivo del estudio. En este sentido, a partir de él, se llevó a cabo una delimitación conceptual de 'marco teórico', de 'conceptualización/definición' y de 'tópico'. También se clarificaron los controles científicos a los que son sometidos los distintos tipos de publicaciones (p.e. artículos, libros, Tesis Doctorales, comunicaciones a Congresos, ponencias, etc.) y el tipo de información que se suele incluir en cada una 
de estas publicaciones. En función de ello, tres jueces multidisciplinares (Psicología Social, Psicología del Trabajo y de las Organizaciones y Psicología Evolutiva y de la Educación) delimitaron los criterios de inclusión y exclusión.

La búsqueda se realizó en SCOPUS, Proquest Central, DIALNET, PsycARTICLES y Web of Science. Se ha optado por estas bases de datos ya que son algunas de las que tienen mayor número de artículos y abogan por el acceso abierto a dicha literatura científica. En ellas se utilizaron los términos de 'employabiliy', 'concept' y 'framework' en el título y resumen. No se restringió el criterio temporal, criterio geográfico ni el idioma de los artículos. Los duplicados se eliminaron. Como criterios de elegibilidad se incluyen sólo artículos de revistas científicas con revisores. Excluimos, pues, aquellos artículos que son monografías, libros, y páginas divulgativas. Respecto al criterio geográfico, se han aceptado todos los artículos. Respecto al criterio de contenido, se han seleccionado solo los artículos que hacen referencia al concepto de empleabilidad o marco teórico de la misma tal y como lo habían delimitado los jueces. Así pues, se han excluido todos los artículos que no hacen referencia al concepto o marco teórico de la empleabilidad; por ejemplo medida de la empleabilidad, programa de intervención, reflexiones sobre su incidencia en el contexto social, etc.

El proceso seguido se presenta en el diagrama de PRISMA (figura 1). En la figura 1 se explicita que se evaluaron inicialmente los títulos y los resúmenes de los estudios. Si estos coincidían con los criterios de inclusión se buscaron y descargaron los textos completos. Posteriormente, se leyeron todos los artículos descargados y se evaluó si el texto del mismo cumplía con cada uno de los criterios de inclusión y no poseía ningún criterio de exclusión previamente mencionados. Para su análisis, se cumplimentó, de cada uno de los artículos, una ficha de contenido. A continuación, se realizó un análisis de contenido y se extrajeron los datos más relevantes de cada artículo organizados en función de las categorías consideradas.

Para analizar y organizar el contenido de los marcos teóricos y las conceptualizaciones de la empleabilidad en orientaciones teóricas, creamos una categorización utilizando los criterios que propone Fox (1981): amplitud, exclusión mutua, consistencia, relevancia, objetividad y lealtad, y productividad. Para ello, propusimos subcategorías que identificaban las premisas teóricas a modo de microaspectos basados en técnicas de codificación inductiva (Llinares-Insa, González-Navarro, Córdoba-Iñesta y Zacarés-González, 2018). Con ellas elaboramos un repertorio que describía la empleabilidad. Para ello tuvimos en cuenta la clasificación de los indicadores de empleabilidad de Llinares, Córdoba y Zacarés (2011). Tres expertos de diferentes disciplinas (Psicología del Trabajo y de las Organizaciones, Psicología Social y Psicología Evolutiva y de la Educación) dieron un nombre a estas similitudes lo cual generó una clasificación de orientaciones teóricas con sus premisas básicas (Moreno, Santos, 
Ramos, Sanz, Fuentes y Del Villar, 2002). Con esta categorización se pretendía asegurar una homogeneidad interna entre las diferentes premisas teóricas clasificados en cada orientación teórica y una homogeneidad externa entre las orientaciones teóricas (Anguera, 1986). Dos jueces independientes codificaron las premisas teóricas de las orientaciones teóricas seleccionadas. La consistencia y fiabilidad de la clasificación de las estrategias de búsqueda de empleo se obtuvieron mediante un acuerdo entre los tres jueces (Krippendorff, 1990, 2013). El valor del Kappa de Cohen superó el 0,99, lo que indica un alto nivel de acuerdo (Altman, 1991).

\section{Figura 1.}

Pasos para la realización de la búsqueda bibliográfica siguiendo la declaración PRISMA

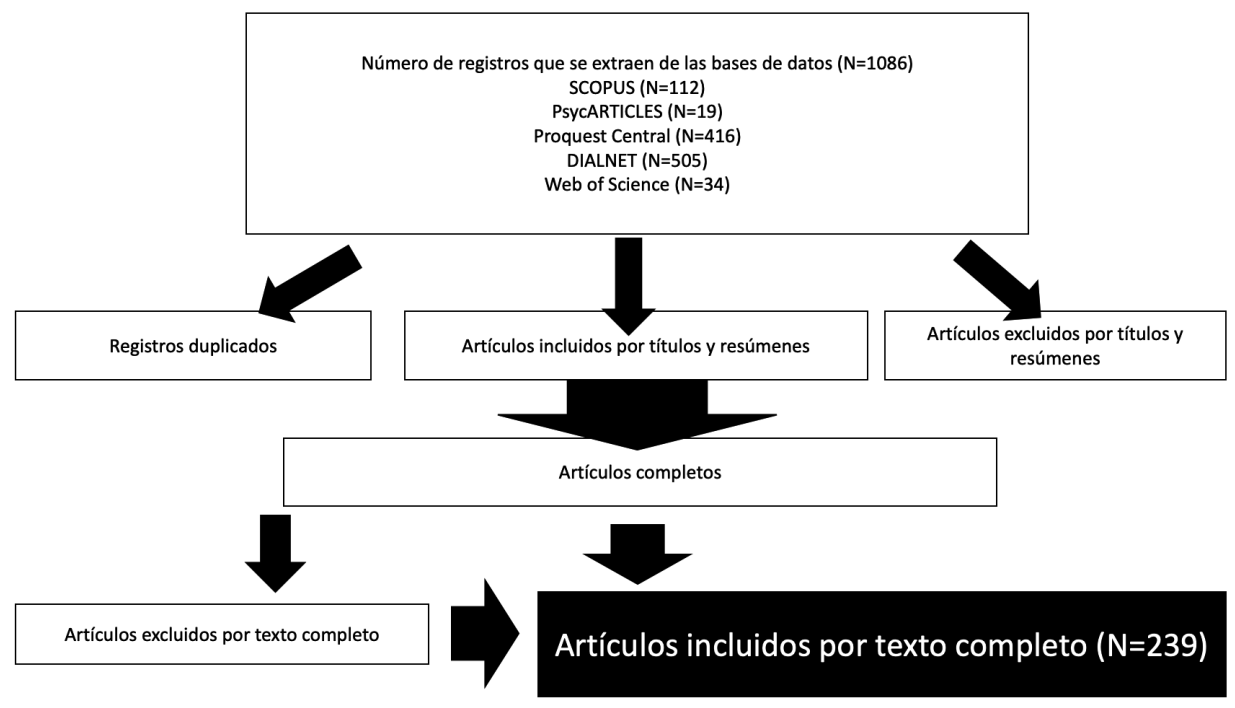

\section{Resultados}

El número de artículos científicos analizados fue de 239. Todos los estudios escogidos tratan el concepto o proponen/presentan un marco teórico sobre empleabilidad. Los tres jueces establecieron dos categorías para articular el contenido de dichos artículos: evolución histórica del término y la estructuración de los marcos teóricos en tres perspectivas teóricas. Así pues, el análisis e integración de los artículos revisados lo hemos organizado en estos bloques. En primer lugar, se presenta la evolución histórica del término. En segundo lugar, las corrientes epistemológicas sobre la empleabilidad. En este segundo apartado se ha analizado la perspectiva individualista de 
mercado empresarial, la perspectiva socio-crítica y el modelo bioecológico sobre la empleabilidad como propuesta integradora.

\subsection{Evolución histórica del término}

La noción de empleabilidad se ha integrado recientemente en el lenguaje, aunque surgió hace décadas (Serrano, 2000). Desde su aparición, ha habido un interés creciente por este concepto debido a los cambios demográficos, organizacionales y la erosión gradual de la seguridad laboral en los países occidentales como resultado de la transición de la sociedad industrial a postindustrial (Clarke, 2008, 2013; Van der Heijde y Van der Heijden, 2006). Así, hasta principios de 1800 el empleo se reducía en esencia a actividades ligadas a las tareas a realizar, continuamente cambiantes, y no a puestos específicos con límites claramente definidos (Bridges, 1994). Con la revolución industrial cambiaron los modelos de empleo, siendo el empleo más estructurado y los roles más definidos. En este contexto era empleable aquel con un conocimiento, habilidades y conductas específicas capaz de demostrar siempre lealtad (Rousseau, 1995). Por otra parte, en los comienzos de la sociedad industrial no se distinguía entre el pobre y el desempleado; ambos se consideraban perezosos y poco motivados para trabajar; por tanto, responsables de su situación (Serrano, 2000). Los cambios sociales propiciaron un análisis del desempleo que promovió el distinguir entre factores individuales (de carácter voluntario) y sociales (de carácter involuntario) (Serrano, 2000).

El estudio de la empleabilidad ha sido objeto de interés para numerosas disciplinas como la administración y dirección empresas, la gestión y desarrollo de recursos humanos, la psicología, las ciencias de la educación y desarrollo profesional (Van der Heijde y Van der Heijden, 2006), etc. Además, se ha utilizado e interpretado de formas muy diversas en función del contexto y en paralelo a los cambios en las carreras profesionales y los modelos de empleo (Clarke, 2008). Es un concepto multidimensional que ha sido popular en tres momentos a lo largo del siglo XX, años 30, 60 y 80, como veremos a continuación. Sin embargo, pocos estudios han intentado integrar las distintas concepciones surgidas a lo largo del siglo XX. De hecho, autores como Rajan, Van Eupen, Chapple y Lane (2000) afirman que la empleabilidad es una de los términos que ha pasado de cliché a jerga sin intermediar una etapa en la que se le dé un significado.

A lo largo del siglo XX estuvieron presentes diversas concepciones de empleabilidad con consecuencias a nivel organizacional (Rentería y Malvezzi, 2008). Sin embargo, pocos estudios han intentado integrar estas diferentes perspectivas. De hecho, 
según Formichella y London (2005) o Van der Heijde y Van der Heijden (2006), aunque el concepto surgió antes, las primeras publicaciones sobre el concepto de empleabilidad datan de la década de los 50, concretamente hacia 1955, y hacían referencia a la preocupación acerca de la posición de las personas desamparadas en el mercado de trabajo, como por ejemplo los minusválidos. Pero no fue hasta los años 90 cuando se estudió de forma empírica (Van der Heijde y Van der Heijden, 2006) y, aunque el término empleabilidad todavía no aparece en todos los diccionarios y libros jurídicos, se ha vuelto popular desde entonces (Bollérot, 2001).

Siguiendo un recorrido histórico por décadas del concepto de empleabilidad, a partir de los años 50 aparecen las versiones "anglosajonas modernas" que incluyen a la Europa continental y particularmente a Francia (Rentería y Malvezzi, 2008). La empleabilidad comenzó a analizarse de manera general como el potencial de un individuo para acceder a un empleo y permanecer en él (Grip, Van Loo y Sanders, 1999).

En los años 60, Gazier $(1998,2001)$ distingue tres tipologías de empleabilidad. La primera es la empleabilidad sociomédica, que emergió en EEUU, Reino Unido, Alemania y otros países, y se refería a la distancia entre las habilidades reales para el empleo de personas con desventaja social, física o mental y los requerimientos del empleo. Se centraba en la limitación y discapacidad, la medición individual en la cual se consideran grados de aptitud física o mental por áreas de desempeño y, finalmente, los problemas de intervención de acuerdo con las deficiencias por compensar. Incluía a médicos y terapeutas de diferentes campos de rehabilitación (Rentería y Malvezzi, 2008).

La segunda tipología Gazier $(1998,2001)$ la denomina empleabilidad de política de mano de obra, fue desarrollada como un intento de extender las discusiones sobre la perspectiva sociomédica a otros grupos desfavorecidos, con el énfasis todavía en la distancia entre las habilidades para el empleo reales y los requerimientos del empleo.

En los 60, en la literatura sociológica francesa, surgió en palabras de Gazier $(1998,2001)$ la empleabilidad fluida que se centró en la demanda y accesibilidad al empleo en economías locales y nacionales. Se definía empleabilidad como la alta probabilidad que tiene una persona que busca trabajo de encontrarlo. Así, se enfatiza la rapidez con que los desempleados encuentran trabajo. La medición se da en función de proporciones por grupos sociales, empleables y no empleables, por la relación de las tasas de desempleo en el mercado de trabajo y por la consideración de las desventajas grupales o individuales de las personas que están o no en el mercado de trabajo (Rentería y Malvezzi, 2008).

De este modo, hasta los años 70 la empleabilidad se centraba en la participación en el empleo y se acompañaba de la flexibilidad de la sociedad. Se consideraba al gobierno como el actor responsable de conseguir pleno empleo. En las últimas décadas 
del siglo XX los cambios que obligaron a las organizaciones a reorganizarse como empresas más flexibles cambiaron el sistema basado en el empleo ('job-based HRM system') al centrado en la persona ('person-related HRM system'). Los nuevos conceptos de producción llevaron a hablar de trabajo en equipo más que de división del trabajo. Estos cambios tuvieron implicaciones sobre el perfil del ideal de la persona que trabaja y los tipos de destrezas que necesitaba. Con ello, el capital humano y los recursos humanos ganaron en importancia (Van der Heijde y Van der Heijden, 2006).

En la década de 1970 la empleabilidad se centraba en una medición de deficiencias de cualificación profesional, movilidad y presentación, revelando la distancia entre características individuales y requerimientos de producción y la aceptabilidad en el mercado de trabajo, lo cual se tradujo en programas de intervención orientados al entrenamiento para la selección y en otros programas complementarios sobre "cómo vestir" o "cómo presentarse" a una entrevista de trabajo (situación que no ha cambiado mucho en los últimos años). En el caso de Estados Unidos esta acepción implicaba un proceso de individualización no problemática en el cual las personas asumen su inclusión o no en el mercado de trabajo (Rentería y Malvezzi, 2008).

A partir de finales de los ańos 70 la perspectiva de empleabilidad apareció en Europa cuando se discutía la capacidad de las instituciones para combatir el desempleo reinante, y éstas descubrieron que existía un gran número de personas con una probabilidad casi nula de reinsertase al mercado laboral (Pérez, 2005). Para Gazier $(1998,2001)$ comenzó el concepto de empleabilidad de rendimiento del mercado laboral, utilizado a nivel internacional. Se centraba en los resultados del mercado laboral conseguidos por la intervención de las políticas de empleo y se medía en términos de los días que la persona estaba empleada, horas trabajadas, tasas de salario y otros resultados del mercado laboral para los individuos que participaban en programas de empleo. La empleabilidad se entendía como la probabilidad de obtener una vacante en un mercado de trabajo específico a partir de los atributos con los que está dotado el buscador de empleo y que son los que le permitían superar los obstáculos que le imponía el mercado (Campos Ríos, 2002).

En las décadas de los años 80 y 90, y como consecuencia de lo acontecido en los años 60 y 70, la empleabilidad pasó a tener una concepción diferente en Estados Unidos (Gazier, 2001) asociada, al igual que en sus inicios, a informaciones estadísticas disponibles sobre el empleo, considerando como indicadores las personas que lo obtenían o estaban ya empleadas, su duración y el salario (Rentería y Malvezzi, 2008). En esta etapa los factores que le dieron una mayor entidad al concepto de empleabilidad incluían: su rol potencial de abordar la inclusión social de los grupos desfavorecidos, una reacción a las consecuencias de los altos niveles de desempleados 
a largo plazo e inactividad y la tendencia hacia nuevos tipos de relaciones entre quien emplea y la persona a emplear (McQuaid y Lindsay, 2005).

Así, a partir de los años 80 la empleabilidad surgió como recurso para explicar la dinámica de empleo, desempleo y la relación de las personas con los mercados de trabajo; presentaba una inmensa variabilidad de significados que se reflejaba en tantas definiciones como usuarios disertaran sobre ello, a diferencia del uso "preciso" de comienzos del siglo XX desde la economía (Rentería y Malvezzi, 2008).

A finales de los 80 surgió el concepto de iniciativa de empleabilidad, que emergió en la literatura sobre el desarrollo de los recursos humanos en Norte América y Europa (HRD) (Gazier, 2001). Reflejaba una aceptación de que el desarrollo de una carrera laboral exitosa requería del desarrollo de destrezas transferibles y la flexibilidad para desempeñar diferentes roles en el trabajo. Se centraba en el individuo y en su responsabilidad para desarrollar destrezas y estar empleado.

Por último, surgió la empleabilidad interactiva desde la perspectiva de Gazier (1998, 2001), que emergió primero en Norte América y desde finales de los 80 a nivel internacional. Se refería a los modelos adoptados a partir de los años 90, particularmente los canadienses, en los cuales la empleabilidad era la capacidad relativa de una persona para obtener un empleo significativo, dada la interacción entre características personales y mercado de trabajo. Este modelo reintrodujo las dimensiones individual y colectiva en las cuales la medición consideraba perfiles, estadísticas, trayectorias y circunstancias en función del mercado de trabajo. En este sentido, las acciones sobre la empleabilidad tenían que ver no solamente con las acciones individuales, sino con las políticas estatales de mercado y con la promoción de oportunidades reales para las personas (Rentería y Malvezzi, 2008). Así, mantenía el énfasis en la iniciativa de la persona pero también reconocía que dependía de la empleabilidad de otros y de las oportunidades, instituciones y reglas que gobiernan el mercado laboral (prioridad colectiva/interactiva). Remarcaba el rol de quien emplea y de la demanda laboral para determinar la empleabilidad. Esto conlleva dos implicaciones: por un lado, centrarse en el desempleado a largo plazo y otros grupos desfavorecidos por las políticas de empleo; por otro lado, centrarse desde los gobiernos occidentales en políticas interventoras para prevenir el desempleo a largo plazo y las desigualdades en el mercado laboral.

Sin embargo, hacia el final del siglo XX los modelos de carrera profesional y empleo cambiaron significativamente al pasar las organizaciones a ser más extensas, con continuas reestructuraciones y reducción de personal en respuesta al aumento de las presiones de la competencia (Cascio, 1993). El "antiguo" contrato psicológico implicaba que el trabajo duro, la lealtad y el compromiso con la organización aseguraban de forma recíproca la seguridad laboral, las oportunidades profesionales, 
el entrenamiento por parte de la compañía y la promoción interna (Baruch, 2001). Por el contrario, el "nuevo" contrato psicológico se centraba más en los elementos transaccionales o "en compromisos específicos, a corto plazo, suponiendo una participación limitada de las partes implicadas" (Morrison y Robinson, 1997).

Aunque por lo general se aceptan estos nuevos modelos, algunos autores se cuestionan si los modelos de carrera profesional y empleo han cambiado en realidad. En cierto sentido, Clarke (2008) observa que el punto de vista actual sobre la empleabilidad parece haber vuelto al modelo pre-industrial, donde el sujeto era el responsable de su empleabilidad. Sin embargo, esta autora considera que se espera que en el siglo XXI haya una continua emergencia de nuevas formas de empleo y nuevas formas de trabajar. Estos cambios señalan la necesidad de continuar investigando sobre el concepto de empleabilidad (Clarke, 2008) porque ésta pasa a ser considerada un componente crucial para conseguir y mantener el empleo (Forrier y Sels, 2003). Junto a ello, los organismos internacionales también resaltan la importancia de la empleabilidad para la reducción de la exclusión social.

\subsection{Las corrientes epistemológicas sobre la empleabilidad}

Para el análisis de las corrientes epistemológicas se llevó a cabo un vaciado de las propuestas teóricas y conceptuales de todos los artículos por medio de una ficha de vaciado. A partir de ellas, se realizó un análisis de contenido. El análisis de contenido dio como resultado tres grandes orientaciones teóricas: orientación individualista, orientación crítica y modelo bioecológico.

\subsubsection{Perspectiva individualista del mercado empresarial}

Es la perspectiva dominante y la más desarrollada (p.e. Andrews y Higson, 2008; Dacre, Qualter y Sewell, 2014; Rothwell, Herbert y Rothwell, 2008). La teoría del capital humano le sirve de base (Keeley, 2007) y su estructura está claramente determinada por los agentes sociales más relevantes implicados y que le sirven de hilo conductor: la persona empleable y la empresa quien emplea.

a) El individuo como responsable de su futuro laboral

Desde esta perspectiva, la persona empleable es el principal protagonista del concepto de empleabilidad. Es la responsable de desarrollar su carrera laboral (Ko- 
valenko y Mortelmans, 2016) y vida profesional (Clarke, 2008). Por tanto, su rol contempla la tarea emergente de adquirir cualidades que la hagan más empleable (Fugate y Knicki, 2008). Como contrapartida, tiene derecho a demandar formación y oportunidades de aprendizaje a la empresa (Montańana, 2005).

En la literatura que mantiene esta perspectiva encontramos dos perfiles de persona empleable que se corresponden con dos modos de entender la 'empleabilidad' (Brown, Hesketh y Williams, 2003): por una parte, la empleabilidad aplicada a la búsqueda del primer empleo (formarse y dotarse de cualidades necesarias para acceder al mercado laboral) y, por otra, la empleabilidad aplicada al mantenimiento o cambio de trabajo en la misma o diferente organización (aumento de la valía de la persona) (Lisbona, Palací y Moriano, 2002).

Con respecto a la primera aproximación, la empleabilidad se refiere a la capacidad que tiene una persona para conseguir y mantener un puesto de trabajo (ver, por ejemplo, Nalawade, More y Bhola, 2019). Podríamos decir que, en concreto, esta primera aproximación se centra en las dificultades que pueden encontrar en la consecución del empleo de colectivos vulnerables. Estos se mantienen, pues, en las capas más pobres de la sociedad (Weinberg, 2004). Esto sucede con grupos poblacionales vulnerables, como los contemplados en la normativa de las empresas de inserción (EI), mujeres, inmigrantes, pertenecientes a las capas desfavorecidas de la sociedad, personas con discapacidad. Puede ocurrir incluso con los empleados de larga duración que, según Weinberg (2004), se tornan dependientes de las políticas sociales. Sus posibilidades de empleabilidad son cada vez menores, ya que van perdiendo sus conocimientos, van disminuyendo los hábitos de trabajo, su capacidad de relacionarse, de adaptarse a los cambios, etc., lo que genera que se vayan profundizando más en su exclusión y sean cada vez menos empleables.

Gordon, Edwars y Reich (1982) afirman que aspectos como la clase social, la educación y la raza afectan a la distribución de las personas en los distintos segmentos del mercado de trabajo (Riquelme y Razquin, 1999). En este sentido, la teoría de la segmentación presenta la empleabilidad vinculada a la estructura social.

Esta perspectiva distingue, pues, entre ser empleable y no empleable. La empleabilidad es concebida como la capacidad para obtener y/o mantener el empleo y/o generar autoempleo (Campos Ríos, 2003; Finn, 2000; Formichella y London, 2005). Esta perspectiva unidimensional etiqueta a la personas como empleables o no empleables en función de su capacidad para conseguir un empleo (Finn, 2000). Pone de manifiesto que son importantes las acciones que haga el sujeto para aumentar dicha capacidad. Esta actividad es un recurso que puede disminuir el desempleo causado por la falta de personal cualificado (Weinberg, 2004). Es por ello que se impone la necesidad de generar actividades individualizadas que aumenten la emplea- 
bilidad de la persona (McQuaid y Lindsay, 2005b). En este sentido se han dirigido la mayor parte de las acciones sociales y es uno de los cuatro pilares de la European Employment Strategy (McQuaid, Green y Danson, 2005). La Comisión Europea pretende el desarrollo del capital humano a través de actividades preventivas para los desempleados (Ramos, 2010).

A pesar de ello, es responsabilidad de la persona fomentar su empleabilidad (Weinberg, 2004). Desde esta perspectiva también se leen discursos que reivindican la importancia de la localización del mercado de trabajo, de aspectos relacionados con los desplazamientos, las actitudes, las conductas empresariales, los requerimientos de las economías locales y otros factores contextuales que impactan en las experiencias del que está buscando empleo (McQuaid y Lindsay, 2005). En definitiva, el sistema productivo, con la empleabilidad como razón discursiva, fundamenta el orden social como sistema de clasificación, jerarquización y valoración de las personas (Prieto, 1999).

La segunda aproximación a la empleabilidad en el enfoque individualista se centra en la necesidad de que los empleados se adapten a las nuevas situaciones del mercado laboral y los nuevos tipos de organización (Formichella y London, 2005; Fugate et al., 2004) y que manejen múltiples identidades (Hall, 2002). La empleabilidad es, pues, la habilidad para obtener, mantener y/o movilizarse hacia otros empleos (Campos Ríos, 2003; Finn, 2000; Formichella y London, 2005; McQuaid et al., 2005) o progresar en la misma organización con el fin de obtener un trabajo que le permita la inclusión social (Fuster, 1999). Así, es considerada como un proceso permanente de adquisición y logro de empleo y una oportunidad de satisfacción profesional y de dirección del aprendizaje y desarrollo (Hind, 2005). Desde una perspectiva más holística se enfatizan junto a las características individuales las condiciones del mercado laboral, sean éstas de demanda y/o de oferta. En definitiva, como proceso se refiere al empleo como el resultado esperado (Van der Heijden y Van der Heijden, 2006). Todo ello supone, en primer lugar, que la persona es responsable de su carrera profesional y, en segundo lugar, que la persona posee habilidades de 'alto rendimiento'.

Que la persona sea responsable de su carrera profesional implica que toda persona es la que acumula destrezas, habilidades, conocimientos, etc. que le permiten optimizar sus competencias y ser más eficaz y eficiente en su trabajo. La persona tiene la responsabilidad de crear su curriculum y es la que tiene la responsabilidad de mejorar su empleabilidad (Fugate et al., 2004). Así, es la persona la que debe gestar su propia competencia (Ducci, 1998). Fugate et al. (2004) utilizan la resiliencia para referirse a las características que fomentan la adaptación de la persona a su puesto de trabajo; dicha adaptación no es pasiva como en el antiguo sistema sino proactiva y para ello es necesario que la persona quiera adaptarse (Hall, 2002). 
La persona adaptable es esperable que afronte con mayor efectividad contextos de incertidumbre que la persona más rígida (Ashford y Taylor, 1990). Su propensión a aprender facilita tanto la creación como el mantenimiento de redes sociales que son centrales en su identificación y consecución de oportunidades laborales. Además, las personas adaptables quieren mejorar sus capacidades, lo cual es fundamental para el capital humano.

La adaptación efectiva y, por tanto, el mejor aprovechamiento del capital humano (y aumento de la competitividad de la empresa) se produce cuando las personas (Ashford y Taylor, 1990): a) obtienen información adecuada del entorno y feedback relativo a su relación con dicho entorno; b) cuentan con ciertas características internas que favorezcan la adaptabilidad y la capacidad de enfrentarse a los retos que conlleva el cambio (Martínez-Cerdá, Torrent-Sellens, González-González y Ficapal-Cusí, 2018); y c) aseguran la movilidad, flexibilidad o libertad de cambio (McQuaid y Lindsay, 2005). Las personas deben desarrollar su empleabilidad a lo largo de la vida y el icono de mayor desarrollo es el estudiante universitario (Conchado y Carot, 2013).

Que la persona posee habilidades de alto rendimiento supone otorgarle habilidades como, por ejemplo, espíritu emprendedor, estar altamente motivado o poseer habilidades metacognitivas, etc. La persona que emplea busca empleados que tengan la capacidad de aportar algo de forma inmediata y a corto plazo antes que individuos que busquen carreras profesionales de larga duración y estables (Clarke, 2008). En este sentido, para este enfoque resulta de suma importancia detectar cuáles son esas cualidades que hacen empleable (Formichella y London, 2005). Sin embargo, la literatura sobre el tema no presenta unicidad en relación a los elementos de la empleabilidad (ver, p.e. Ferns, Dawson y Howitt, 2019; Finch, Peacock, Levallet y Foster, 2015; Mohamad, Jamaludin, Zawaei y Wan Hanafi, 2018). En su mayor parte, la literatura revela que es multidimensional (Yizhong, Lin, Baranchenko, Lau, Yukhanaev y Lu, 2017). La mayoría de los estudios analizan el nivel educativo (Formichella y London, 2005; Van der Heijden y Van der Heijden, 2006). Por ejemplo, surgen muchas publicaciones sobre la medida de la empleabilidad de los estudiantes (p.e. Koloba, 2017) y sobre cómo desarrollar la empleabilidad por parte de la institución educativa (p.e. Ciesielkiewicz, 2019) y/o universidad (p.e. Bates, Hayes, Walker y Marchesi, 2018; Clarke, 2018; Räty, Komulainen, Harvorsén, Nieminen y Korhonen, 2018). Otros autores destacan aspectos como la capacidad de comunicación (Sharma y Shivakumar, 2019), de manejar procesos de organización, de trabajar en equipo (Bharathi, 2016), el autodesarrollo del individuo (planificación de su vida profesional, actitud proactiva) (Fuster, 1999), el aprender a aprender (Martínez-Cerdá y Torrent-Sellens, 2017; Rentería y Malvezzi, 2008), etc. 
Entre las clasificaciones con fundamentación teórica cabe resaltar la de Van der Heijden y Van der Heijden (2006), que presenta una clasificación elaborada y bien fundamentada en la que la empleabilidad está compuesta por cinco competencias: la experiencia ocupacional, la anticipación y optimización, la flexibilidad personal, el sentido corporativo y el equilibrio. Por su parte, Fugate et al. (2004) distingue las siguientes dimensiones: la identidad profesional, la adaptabilidad personal y el capital humano y social. Estas dimensiones no operan de forma independiente sino de forma global. En el mismo sentido, McQuaid y Lindsay (2005) proponen, desde un enfoque amplio, una clasificación de sus componentes en factores individuales, circunstancias personales y factores externos. El estudio propuesto por Malaysian Engineering Employability Skills (MEES) destaca tres componentes principales de la empleabilidad: atributos personales, habilidades personales y conocimiento (Nisha y Rajasekaran, 2018)

La percepción del empleador en cuanto a los componentes de la empleabilidad también es otro de los tópicos más analizados desde la literatura científica Desde el punto de vista de la persona que emplea parece que algunas de las competencias más valoradas son, por ejemplo, los recursos, la información y la tecnología (Tejan y Sabil, 2019).

Junto a ello, otros autores también tienen en cuenta aspectos como: edad, tipo de institución educativa, forma de estudio, actualización o recualificación, experiencia previa, etnia, género y clase social (Harvey, 2001). Así pues, tal y como señalan Van der Heijden y Van der Heijden (2006), no es un constructo unidimensional sino compuesto por factores muy diversos; para ser considerado empleable no todos ellos tienen que cumplirse, o sí, depende de la perspectiva desde la que nos situemos (Van der Heijden y Van der Heijden., 2006).

En definitiva, este panorama tan abierto muestra que la empleabilidad abarca diferentes competencias (Formichella y London, 2005) lo que nos lleva al concepto de 'competencia'. La cuestión de las competencias es uno de los principales mediadores que se asume desde la empleabilidad; el 'saber hacer en un contexto' se une al 'preferir hacer' y al 'hacer con eficacia' (Rentería y Malvezzi, 2008) en la empleabilidad.

Ahora bien, por qué surge el concepto de empleabilidad. La mayor parte de los autores indican que la empleabilidad surge como alternativa a la seguridad en el empleo (Baltanás, 1999; Bernstrom et al., 2019) y por la tendencia hacia nuevos tipos de relaciones entre el empleador y el empleado (McQuaid y Lindsay, 2005).

Se convierte en el nuevo contrato psicológico entre la empresa y el empleado donde los dos son responsables de mantener la situación de empleo (Lisbona et al., 2002). Así pues, la gestión y el perfeccionamiento de los recursos humanos pasa a ser un factor determinante tanto de la competitividad de las empresas como de la 
seguridad en el empleo (Ducci, 1998; Fugate et al., 2004. En este sentido, resulta útil distinguir entre el significado de búsqueda de empleo y empleabilidad. Para Kanfer, Wanberg y Kantrowitz (2001), la búsqueda de empleo es "un patrón de acción propositivo y deliberado que empieza con la identificación y compromiso de perseguir una meta laboral. Dicha meta activa la conducta de búsqueda para alcanzarla”. Desde ese planteamiento, la empleabilidad es una estrategia para sustituir la seguridad perdida y el mecanismo para aumentar el compromiso del empleado hacia su empresa (Lisbona et al., 2002). La persona empleada tiene que rentabilizar su curriculum a lo largo de toda su vida laboral. Así, la autogestión de su carrera es la clave del éxito (Baruch, 2001).

Desde esta perspectiva se afirma que las personas perciben esta mezcla entre libertad y responsabilidad de sus carreras laborales como preferible a las experiencias de organización de carrera y seguridad en el empleo (Bagshaw, 1997; Clarke, 2008). Así pues, ya no es la organización la que dirige hacia dónde van los individuos, sino que cada persona toma el control de su carrera y debe desarrollar su empleabilidad por medio del aprendizaje (Peter y Gómez, 2019). Desde este punto de vista, la alta empleabilidad puede amortiguar y prevenir el desempleo (McArdle, Waters, Briscoe y Hall, 2007).

b) La empresa como agente social de la empleabilidad

Junto con los factores individuales, esta perspectiva también destaca la importancia de factores contextuales como los que se refieren a la situación del mercado laboral (McQuaid y Lindsay, 2005). Así, por ejemplo, las prácticas universitarias conectan al joven con el mundo empresarial (Caballero, Vázquez y Quintás, 2015). Estas prácticas también son relevantes porque el hecho de cursar los estudios universitarios no genera empleabilidad. Para ello se necesitan las prácticas pre-laborales (Tejan y Sabil, 2019). Esto es relevante ya que desde la perspectiva individualista la empleabilidad beneficia a la persona y a la empresa (Yorke, 2006). La empresa debe contratar empleados con alto grado de empleabilidad y, por eso, actualmente, se ha desarrollado una línea de trabajos que intentan operacionalizarla y están dando como resultado diversos cuestionarios que miden la empleabilidad (ver por ejemplo Singh, Chawla, Agarwal y Desai, 2016; Van der Heijden y Van der Heijden, 2006; Vargas, Sánchez-Queija, Rothwell y Parra, 2018). Paralelamente, también se acentúa la obligación de la empresa de proporcionar oportunidades para el desarrollo (Baruch, 2001) y de desarrollar y mantener las habilidades generales avanzadas (Clarke y Patrickson, 2008), esto es, de generar empleabilidad que posibilite la reconversión 
de los recursos humanos y poder afrontar los cambios en el trabajo y en el mercado laboral (Salanova y Llorens, 2006). En definitiva, la empresa tiene la obligación de generar empleabilidad, de modo que si una persona pierde el empleo no tenga dificultades para encontrar otro (Van Buren, 2003).

Además, aunque el papel fundamental en la empleabilidad lo tiene el sujeto (Fugate et al., 2004) y, en parte, la empresa, el Estado y la sociedad en general también tienen parte de responsabilidad (Gamboa, Gracia, Ripoll y Peiró, 2007). Olssen y Perters (2005) plantean que en el sistema neoliberal el estado tiene la función de crear las concisiones, leyes e instituciones que posibiliten su libertad. Así, el Estado tiene la función de prevenir y tratar de corregir la empleabilidad o desarrollarla (que genera desigualdad y exclusión social) por medio de la educación. La educación formal es su principal instrumento (Campos Ríos, 2003; Formichella y London, 2005). La educación no formal también está encargada de desarrollar la empleabilidad. Al mismo tiempo, la educación informal tiene que generar empleabilidad y emprendimiento (Formichella y London, 2005). Por lo tanto, el grado de empleabilidad dependerá de los atributos y habilidades, pero también de otras consideraciones extraeconómicas (políticas, raciales, institucionales).

\subsubsection{Perspectiva socio-critica sobre la empleabilidad}

La perspectiva crítica, también denominada teoría del conflicto (Brown et al., 2003), concibe los cambios económicos y del mundo laboral de forma diferente a la perspectiva presentada anteriormente. La empleabilidad es considerada como la disposición requerida para funcionar eficazmente en economías que cambian rápidamente y que se hallan sujetas a una fuerte presión competitiva (Thieme, 2007). Esta perspectiva presenta un discurso crítico hacia la aparente racionalidad del mercado (Brunet y Pastor, 2003).

Afirman que la empleabilidad es una construcción social (Castillo, 1999) que se inicia en las diferencias de clase y las mantiene (Brunet y Pastor, 2003; Reid, 2015). Desde esta perspectiva, las políticas ocupacionales son consideradas ineficaces. La acción pública opta por liberar y desregular el mercado laboral y esto genera y mantiene nuevas desigualdades. Las políticas de formación profesional y empleo ocultan factores socio-económicos como el paro, la precariedad y la amenaza de despido. En esa línea, se señala que tales políticas se centran en la igualdad de oportunidades y de formación de todo ciudadano aunque las sociedades no practican la equidad social (Brunet y Pastor, 2003). Es en este marco en el que hay que entender la crítica al concepto de empleabilidad. 
De esta forma, la empleabilidad se ha situado en el plano de lo simbólico, de las percepciones, significados y discursos reelaborados por los grupos sociales (Carrieri y Sarsur, 2002). Un discurso que desde esta perspectiva crítica se pretende desvelar es sus implicaciones para mantener el status quo de determinadas clases sociales $y$, para ello, usan la individualización de los beneficios/recompensas en función de la inversión realizada en educación.

a) La empleabilidad como justificación de la desigualdad

La perspectiva crítica plantea que, por una parte, se atribuye al individuo el éxito o fracaso de su carrera laboral sin tener en cuenta las competencias que posee y las que demandan los puestos de trabajo. Por otra parte, se parte de la creencia que el mercado laboral es un dato dado y homogéneo y que son las competencias las que hacen que pueda participar en él (Martín y Spinoza, 2005).

En sus inicios la acción de la empleabilidad estaba restringida al momento de la contratación (por ejemplo, la teoría del 'puerto de entrada', ver Piore, 1995). Posteriormente, se señala que el discurso dominante en el ámbito empresarial sobre la empleabilidad se basa en la representación social de que para progresar en el mundo laboral los ciudadanos deben tener un proyecto profesional de vida, deben poder formularlo y llevarlo a cabo (Brunet y Pastor, 2003). La implicación de esto supone la justificación de las diferentes posiciones sociales de cada cual desde un argumento meritocrático: quien no triunfa es porque no ha seguido dicho proyecto o porque no lo ha desarrollado. De esta forma, se ha forjado una nueva cultura centrada sobre los conceptos de mercado, individuo, eficiencia y competitividad (Brunet y Pastor, 2003) que tiene en su inicio y fin la empleabilidad y que responsabiliza al individuo de su logro. Este concepto favorece la lectura del desempleo como 'carencia' (individual) y no como riesgo (social) (Serrano, 2000). Este intento de legitimar las desigualdades en el mercado laboral sustituye al principio de la selección escolar, ya que la igualdad de oportunidades se percibe como garantizada (Pérez, 2018), se amplía a las desigualdades en los salarios y pasa a ser considerada una táctica de poder mediante la cual se reproducen las desigualdades ocupacionales (Brown et al., 2003). Desde esta perspectiva, la referencia del concepto analizado es siempre la persona que es empleable por la organización. De esta forma, se dejan invisibles otras dinámicas de inclusión/exclusión social y laboral, se quita la responsabilidad a la sociedad y al mercado de trabajo. Así, junto con la persona que accede al empleo o que quiere mantenerlo y la empresa se destaca la importancia de los políticos, el Estado y los colectivos con incidencia social como los gremios, sindicatos, etc. (Rentería y Malvezzi, 2008). 
Por tanto, el sistema formal enmascara la dinámica de competitividad impuesta a los estudiantes para alcanzar niveles educativos que les permitan desarrollar competencias necesarias para entrar en el mercado de trabajo (Rentería y Malvezzi, 2008); es un planteamiento claramente selectivo. Para ello, desde la infancia se invierte tiempo, esfuerzo y capacidades, sin embargo, no está garantizada ni la cualificación, ni el acceso al trabajo (Brown y Hesketh, 2004). Arrow (1972), en la teoría del filtro, señala que la educación no contribuye directamente al crecimiento de la productividad de los individuos, pero sirve como medio clasificador de las personas. Bourdieu y Passeron (1977) afirman que el sistema educativo contribuye a reproducir y legitimar la estructura social al distribuir a los individuos en clases homogéneas y diferentes entre sí.

Así, las políticas educativas y los programas de recualificación y desarrollo de la empleabilidad se presentan con el objetivo de fomentar el desarrollo de la competitividad (Torres, 2000), los discursos sobre la selección de los mejores se asocian a perfiles de competencias y cualidades (Rentería, 2005) y dividen a la población entre personas con alta y baja empleabilidad manteniendo, así, discursos hegemónicos. Brown y Hesketh (2004) explicitan que es 'la guerra por talentos' y del 'capital reputacional', aunque es una 'guerra' ficticia. En realidad, tal y como afirman Rentería y Malvezzi (2008), esta cuestión deja abiertos temas como la necesidad de analizar cómo se produce la selección de los que tienen mejor empleabilidad, o la discusión sobre cómo ser realmente considerados visibles y de interés por los mercados de trabajo en los cuales el sujeto debe insertarse (Enríquez y Rentería, 2007).

Si desde la perspectiva individualista se daba por supuesta la responsabilidad del individuo en su inclusión en el mercado de trabajo, desde la perspectiva crítica se señala que ésta realmente depende de las necesidades del mercado, las preferencias de la persona que emplea y las características del puesto (Rentería y Malvezzi, 2008). Se afirma que la desigual distribución del empleo no es debida a las diferencias individuales sino a la propia distribución desigual del mismo que reproduce jerarquías sociales en tanto que es producto de la lógica de jerarquización social, así como la desigual distribución de formación en las empresas. Brunet y Pastor (2003) afirman que es en los análisis de los procesos de asignación de salarios y en los procesos de selección y reclutamiento de los individuos donde se fundamenta la existencia de una estructura productiva. Es la estructura productiva la que impone condiciones de desigualdad fundamentadas en las características individuales desde el discurso social, pero sin vinculación a ellas, en una visión general del proceso. Esto se observa en la homogeneidad de los sectores actuales de excluidos laborales: grupos de edad, mujeres, emigrantes, personas con poco nivel de estudios y cualificación (Tezanos, 2001). 
b) La empleabilidad como complemento de las políticas de flexibilización del mercado de trabajo

Las definiciones de la empleabilidad de carácter mercantil consideran a la persona como sujeto en un contexto dentro del cual es un nómada que enfrenta cambios culturales, de identidad, de exigencia de competencias y es desafiado a vivir como gerente de sí mismo (Castel, 2000). Así, podemos señalar que una segunda consecuencia de considerar a la persona como responsable de su empleabilidad es la transferencia de responsabilidades sobre la situación laboral de las personas y sobre la inclusión/ exclusión del mercado laboral desde agencias sociales como la empresa o el Estado (Carrieri y Sarsur, 2002; Hirata, 1997).

La perspectiva crítica pone de manifiesto que resulta incoherente la construcción de un proyecto laboral profesional homogéneo a partir de trayectorias caóticas de vida laboral. Su formulación permite reducir la responsabilidad de las empresas sobre las condiciones laborales (sobre todo de estabilidad, seguridad y desarrollo), aumentando la flexibilidad en términos de a quién emplearán y bajo qué condiciones. Demuestran que no se ha generado independencia, sino mayores diferencias de fuerzas a favor de la persona que emplea (Legge, 2005). El dominio que se ejerce sobre la persona no se concreta en términos de alienación sino en sobreidentificación del asalariado con su tarea y con la empresa; los límites entre la vida laboral, familiar y social quedan difusos y vuelve vulnerable a la persona y a relegar la vida social y familiar a la vida laboral por miedo a la desocupación (Pérez, 2018). La empleabilidad y la responsabilidad de la adquisición y mantenimiento del empleo es una obligación, y debe ser una forma de predisposición permanente de implicación en el trabajo, de interiorización de la cultura de la empresa (Brunet y Pastor, 2003) y la elección en los conflictos intrapersonales de la dimensión laboral. La empleabilidad incluye en su seno y, por ende, la representación social de la persona que trabaja muy bien, la posesión de competencias que le hacen vivir por y para la empresa.

Además, el concepto de empleabilidad introduce nuevos modos de control social: el autocontrol (Sennet, 2010). Así, el compromiso de los poderes públicos se diluye y se le otorga a la familia y al individuo el honor y la responsabilidad de ser el máximo responsable de su trayectoria laboral (Brunet y Pastor, 2003).

En tercer lugar, la perspectiva individualista cita a los profesionales bien capacitados o en posiciones directivas como modelos de carreras autogestionadas (Currie, 2006). Sin embargo, como señalan Bloch y Bates (1995), gran parte de la población no desea la autogestión de su carrera. Desde luego, aunque entre las personas de reciente titulación, ya socializados en esta nueva forma de comprender la carrera profesional, se perciba como una oportunidad, no sucede lo mismo con los indivi- 
duos socializados en la cultura tradicional del trabajo; y mucho menos cuando nos referimos a las personas que trabajan en sectores menos cualificados. Es en estos casos cuando el concepto de empleabilidad se muestra más equívoco: la precarización del mercado de trabajo y la descualificación que se produce en muchos sectores no nos permite hablar, sin caer en serias contradicciones, del desarrollo de la empleabilidad como factor de estabilidad laboral y menos de desarrollo profesional. En un mercado 'secundario' caracterizado por trabajos con estatus diferentes, precarios o inestables, salarios bajos, malas condiciones de trabajo, baja calificación y sometidos a despidos es difícil construir un proyecto profesional homogéneo. Trayectorias caóticas no dan paso a proyectos estructurados de carreras profesionales preprogramadas y planificadas (Pérez, 2018). La autogestión supone, en definitiva, la sumisión a las necesidades de las empresas a razón de las necesidades del mercado, oculta la precariedad y las escasas posibilidades de desarrollo profesional e implica inseguridad en el empleo (Lugo y Alcántara, 2017).

En cuarto lugar, la planificación del futuro profesional incluye, en una primera fase, el empleo precario (Pérez, 2018). De esta forma se justifica la existencia del mismo y el paso provisional de todas aquellas personas con proyecto de vida laboral. Se legitima también la falta de salida del primer escalón del proyecto profesional por las características del individuo, no del sistema. Se lleva a cabo un acercamiento que culpabiliza a la víctima (Hillage y Pollard, 1998).

La empleabilidad se asocia con las características del sujeto que le permiten ir afrontando las etapas del desarrollo de una carrera profesional (Fugate et al., 2004). El problema surge en la medida en que la contratación, el mantenimiento del puesto y la posición ocupada no depende de la persona. Hay muchas dinámicas que escapan del control individual del sujeto y, por tanto, de su empleabilidad (Enríquez y Rentería, 2007). Así pues, no se puede concluir la existencia de una relación clara entre empleabilidad y empleo (Clarke y Patrickson, 2008). Los datos muestran que ser empleable no es garantía de convertirse en empleado en el tipo de trabajo deseado y con las condiciones necesarias (McQuaid y Lindsay, 2005). Sin embargo, esa relación es la base de los discursos mercantiles sobre la empleabilidad.

En conclusión, podemos decir que la empleabilidad es, desde esta perspectiva crítica, una cuestión social (Bollérot, 2001). Permite legitimar las diferencias sociales y laborales y contribuye a la reproducción social facilitando las prácticas flexibilizadoras del mercado de trabajo. Además, permite que los problemas sociales se conciban y analicen como problemas personales y no sociales (Brunet y Pastor, 2003). 


\subsubsection{El modelo bioecológico de la empleabilidad: una propuesta integradora desde la psicología}

La aproximación bioecológica de la empleabilidad (Llinares-Insa, Zacarés y Córdoba, 2016) parte de la consideración de la empleabilidad como una construcción social resultado de la interacción recíproca entre un organismo humano biopsicológico activo y en desarrollo y las personas, objetos y símbolos de su ambiente externo que tienen que ver con la adquisición y mantenimiento del empleo. Así pues, esta perspectiva parte de la idea de que la empleabilidad está relacionada con la adquisición y mantenimiento del empleo, es un fenómeno socialmente construido y debe ser entendida como un proceso vinculado a la persona que se construye en su historia individual y social. Pasamos a analizar cada premisa por separado.

Premisa 1: La empleabilidad está relacionada con la adquisición y mantenimiento del empleo

La empleabilidad está relacionada con la adquisición y mantenimiento del empleo, tal y como indica el contenido de la representación social del término y múltiples definiciones del concepto (por ejemplo, Cabo, Pagán y Nieto, 2008; Campos. Rueda, Martins, Mancini, Ghiraldellu y Fumache, 2003; Clarke, 2008; McQuaid y Lindsay, 2005; Nauta et al., 2009; Rothwell y Arnold 2007; Thijssen et al., 2008). Eso hace que el empleo se convierta en función, objetivo y modo de evaluación; además de permitir que se distinga entre ser o no empleable y los grados de empleabilidad. Así pues, genera una clasificación de la persona y una posible categorización social.

Premisa 2: La empleabilidad es un fenómeno socialmente construido

La empleabilidad como construcción social requiere su inscripción en un sistema social concreto y supone que una persona no es inempleable en tanto no se le reconozca como tal. Así pues, la empleabilidad no existe en nuestra dotación genética, sino que es el producto de una regulación social y de las relaciones entre grupos sociales. Afecta al individuo de modo diferente según las condiciones coyunturales y sistemáticas y, especialmente, en función de la pertenencia a determinados grupos sociales.

La reacción social con una persona considerada inempleable conlleva la devaluación del estatus de la persona estigmatizada. Los estigmatizadores consideran que las 
diferentes atribuciones que le hacen al estigmatizado son fruto de las características de las personas a las que se les estigmatiza y no de un conocimiento construido socialmente. Este estereotipo es interiorizado de tal forma que tiende a ser utilizado inconscientemente en la interacción social (Banaji y Greenwald, 1994).

Además, también es cierto que un factor importante de la no erradicación de la empleabilidad una vez genera la estigmatización se debe a los beneficios personales y sociales que genera. Una de ellas es la justificación de las desigualdades sociales. Con ella se pretende, pues, legitimar las diferencias entre clases sociales. Los teóricos del conflicto entre clases postulan que en todas las sociedades hay desigualdad social y que es la gente que detenta el poder la que tiende a estigmatizar a los que no lo poseen para justificar las diferencias sociales (Jost y Banaji, 1994). Desde este grupo de teorías se argumenta que las desigualdades sociales deben ser legitimadas si se quiere reducir el conflicto intergrupal. Para ello se intenta crear consenso en la ideología que promueve la superioridad de un grupo. Crandall (1994) expone que el sistema de justificación de esta ideología es la tendencia a responsabilizar a la persona de su propia situación y de atribuir su situación a factores controlables: cada uno ocupa un lugar social determinado en función de sus capacidades y de su esfuerzo. De esta forma se naturaliza la explicación de las desigualdades y se vuelve instrumento que legitima la discriminación.

Premisa 3: La empleabilidad debe ser entendida como un proceso vinculado a la persona que se construye en la historia individual y social

Esta premisa parte de la comprensión de la empleabilidad tomando como base el modelo bioecológico de Bronfenbrenner (Bronfenbrenner, 2005). Desde este modelo se parte de la consideración de que la empleabilidad se construye por las variables individuales, pero también relacionales. En concreto, Bronfenbrenner y Morris (1998) proponen que "la forma, la fuerza, el contenido y la dirección" de los procesos proximales (de la empleabilidad) varían sistemáticamente como función conjunta de, entre otras, las características de la persona que se evalúa, las características del ambiente, tanto inmediato como más remoto en el que tiene lugar el desarrollo (desde el nivel del micro- al del macrosistema) y las continuidades y cambios ocurridos en el tiempo a lo largo del curso vital, además del período histórico en el que se desarrolla la empleabilidad.

Así, la persona se convierte en el punto de partida del análisis de la empleabilidad. La persona es considerada sujeto activo e intencional como un polo de la interacción ya que construye significados, tiene intenciones, interpreta situaciones, etc. 
De la persona hay tres tipos de características que resultan interesantes (Bronfenbrenner y Morris, 2006) y que deben ser tenidos en cuenta en el análisis de la empleabilidad: a) las fuerzas o disposiciones como, por ejemplo, la impulsividad, la falta de interés o la timidez, la curiosidad, la tendencia a iniciar e implicarse en actividades solitarias o grupales, la disposición a diferir la gratificación en el logro de metas, etc.; b) los recursos o elementos positivos y negativos de carácter biopsicológico que inciden en la capacidad del sujeto para implicarse de manera efectiva en los procesos proximales como, por ejemplo, habilidades, conocimiento y experiencia, los defectos genéticos o físicos, las de enfermedad crónica, etc.; y c) las demandas o características de la persona que afectan al modo en que los demás reaccionan ante ella como, por ejemplo, la apariencia física, los factores sociodemográficos de edad, género y grupo étnico (estos factores no sólo son únicamente características físicas de las personas, sino que ya las sitúan en particulares nichos ecológicos que definen su lugar en la sociedad).

De todos estos elementos destacados se desprende que la empleabilidad es una competencia que es aprendida y que su desarrollo en la persona la vincula a una experiencia social concreta. La importancia del ambiente resulta, pues, clave en este proceso. Desde las teorías de la socialización se pone de manifiesto el papel determinante del proceso de socialización y la importancia de los contenidos culturales que se transmiten en él. En concreto, se afirma que en ambientes desaventajados y desorganizados, el impacto de los procesos proximales será mayor sobre aquellos resultados evolutivos que reflejen una disfunción evolutiva (Bronfenbrenner y Morris, 1998). Así, el desarrollo de la persona en ambientes aventajados y estables que transmiten contenidos acordes con los requisitos y parámetros impuestos por la estructura económica, política, socio-cultural y tecnológica tienen mayor probabilidad de aumentar la competencia funcional de la persona y, por tanto, la empleabilidad. Es por ello que, para la evaluación de la empleabilidad, resulta relevante conocer los diferentes componentes de la socialización y cómo estos inciden en su proceso de desarrollo. Así, los aspectos como la privación de oportunidades de acceso a los bienes y recursos de la comunidad, la privación cultural, las condiciones de vida pésimas y el desfase entre los objetivos y las expectativas que la sociedad exige y las posibilidades reales que ofrecen al individuo, la posición de su grupo cultural en la estructura social y de las interacciones que establece con los demás, etc. son los que definen la inempleabilidad; ésta no es, pues, una cuestión genética sino que es fruto de una relación social conflictiva (Aceña y Caparrós, 2018).

Por otra parte, la empleabilidad debe ser entendida como un proceso que se construye en la historia individual y grupal. El contexto ambiental es concebido como un conjunto de estructuras anidadas una dentro de otra que circundan a la persona de 
la que se evalúa su empleabilidad (Bronfenbrenner, 1987). El concepto de empleabilidad, pues, es dinámico y su visión y evolución se concibe desde unos contextos específicos (micro, meso, exo y macrosistema) que la condicionan y a los que condiciona. En concreto, se señala que:

- La empleabilidad está mediatizada por la interacción de la persona con su contexto inmediato. Se debe atender, por ejemplo, al ajuste de la persona-puesto de trabajo, al apoyo social recibido por parte de todos los entornos en los que está inmersa la persona, se debe tener en cuenta el acceso de la persona a los recursos (poder acceder a una vivienda digna, acceso a recursos de apoyo financiero, acceso a redes de apoyo, ...), etc.

- La empleabilidad nos informa de la repercusión de la interacción de los contextos inmediatos. Así pues, por ejemplo, las circunstancias personales como el cuidado de los hijos determinan la evaluación de la empleabilidad.

- La empleabilidad debe contemplarse desde los contextos que la afectan, pero también sobre los que no puede actuar y se enmarca en un contexto social, político y laboral concreto. Las nuevas condiciones económicas del neoliberalismo y los cambios que generan en el resto de sistemas hacen que la empleabilidad sea un elemento relevante en las relaciones labores. Esto transforma las dinámicas organizacionales y cambia algunas de las aproximaciones clásicas al estudio de las organizaciones e introduce nuevas formas de carrera laboral.

Las características del marco económico, político, socio-cultural y tecnológico, con sus cambios y variaciones, alteran y modifican los requisitos y parámetros de la empleabilidad. Así se introduce la importancia de los aspectos económicos (como los nuevos modos de contratación, la dificultades de inserción laboral en el mercado reglado, las demandas del mercado laboral, inestabilidad y fragmentación del empleo, etc.), políticos (la relación del mundo del trabajo con las políticas públicas, las políticas de ocupabilidad, etc.), socio-culturales (las representaciones sociales y la cultura del trabajo que se transmiten en los medios de comunicación, el flujo de información que circula sobre la comprensión de las situaciones, la relación entre las redes sociales y el empleo, la nueva forma de concebir el empleo y todos los presupuestos teóricos sobre los que se sustentan, etc.) y tecnológico (internet, informática, cambios en los procesos de trabajo, etc.) para el análisis y la evaluación de la empleabilidad. 


\section{Discusión y conclusiones}

La globalización de los mercados ha generado grandes cambios en el mundo laboral (Valero, 2019). En Espańa, el perfil de las personas que trabajan ha cambiado al igual que sus derechos laborales, la temporalidad y precariedad de los contratos (Arestis, Ferreiro y Gómez, 2020). Sin embargo, el efecto perverso de estos cambios no ha tenido las mismas consecuencias en todos los colectivos. En los grupos vulnerables, las últimas reformas laborales han tenido un efecto todavía más grabe. En este contexto resultan clave los agentes sociales que pretenden la inclusión laboral de los colectivos en riesgo de exclusión social. Sin embargo, la empleabilidad, como se ha observado en esta revisión sistemática, se ha entendido y medido de diferentes formas (Römgens, Scoupe y Beausaert, 2019). De este modo, los agentes sociales como, por ejemplo, los trabajadores de acompañamiento (PTA) de las empresas de inserción (EI), deben seguir trabajando en pro del fomento de la empleabilidad desde una única mirada. En este sentido, es necesario un análisis de la literatura sobre el tema que sistematice y ordene las orientaciones teóricas, sus bases conceptuales, sus indicadores y medidas de evaluación.

Este trabajo ofrece una revisión sistemática sobre el concepto de empleabilidad y los marcos teóricos desarrollados para su conocimiento, identificando sus beneficios y sus barreras. De esta forma, se pretende dar a conocer los distintos escenarios que envuelven a la empleabilidad y le dan sentido y significado. En esta revisión, hemos podido ofrecer el recorrido evolutivo sobre el significado de la empleabilidad. Hemos presentado el análisis de la empleabilidad desde las distintas disciplinas dotando de cientificidad, veracidad y objetividad a la representación social del término. Hemos destacado algunos estudios que hacen una propuesta fundamentada sobre el concepto de empleabilidad, como por ejemplo los modelos teóricos presentados por Álvarez-González et al. (2017), Clarke (2018), Fugate et al. (2004), McQuaid y Lindsay (2005), Thijssen y Van der Heijden (2003), Tijssen et al. (2008), Van der Heijde y Van der Heijden (2006) y que han servido de base a estudios posteriores (ver, por ejemplo, Nauta et al., 2008; Rae, 2007). Sin embargo, ninguno de estos estudios proporciona un modelo integrado que pueda dar respuesta a todos sus usos. Para dar respuesta a esta demanda surgió el Modelo Bioecológico de la Empleabilidad propuesto por Llinares-Insa, Zacarés y Córdoba (2016). Este modelo se caracterizaba por considerar la empleabilidad como una categoría histórica construida socialmente y, como tal, condicionada por factores socio-históricos e institucionales. Desde este modelo se acentúa la importancia de las producciones colectivas y compartidas que resultan de la vida en común y que le dotan de cierto dinamismo. Este enfoque parte de la importancia de los elementos socio-estructurales en la génesis del fenómeno, 
aunque todavía da paso más, y se centra en la interacción individuo-sociedad. Considera que la empleabilidad y sus consecuencias se enmarcan dentro de la génesis de la construcción de la realidad social de la que todos somos responsables. Así pues, el conflicto fundamental que implica este concepto en el terreno del análisis social de las relaciones laborales se pone de manifiesto a través de dos grandes perspectivas teóricas: la perspectiva que incide en los factores individuales de la empleabilidad y la perspectiva socio-crítica.

Como resultado de esta revisión, podemos afirmar que el neoliberalismo utiliza el concepto de empleabilidad como clave y motor del empleo. Ante ello, surgen múltiples y variadas aproximaciones que pueden estructurarse en tres corrientes: un marco que se centra en la responsabilidad individual del individuo, otro marco que se centra en hacer una crítica de todas las premisas de la corriente anterior y, en tercer lugar, una aproximación integrativa de la empleabilidad a la que se denomina Modelo Bioecológico de la Empleabilidad (MBE). Esta última conceptualización recoge la complejidad de todos los tipos de colectivos y situaciones en las que se emplea el término. Así pues, proporciona un significado de la empleabilidad que le permite analizar la empleabilidad tanto de un joven estudiante universitario como de un adulto en una empresa de inserción social. También permite el análisis de la empleabilidad que se podría hacer desde los sindicatos o cuando se argumenta sobre los usos legitimadores del orden social, desde las políticas estatales y europeas que pretenden el aumento de la ocupabilidad, e incluso desde las concreciones empresariales sobre las demandas a los empleados, etc.

Junto a ello, en esta revisión sistemática también se ha presentado cómo la empleabilidad, bajo el halo de la objetividad que la envuelve, es la expresión de una sociedad conflictiva debido a las desigualdades sociales y laborales (Serrano, 2000; Van der Heijde y Van der Heijden, 2006). Es más, su uso no sólo fundamenta el orden social como sistema de clasificación, jerarquización y valoración de las personas, sino que es el eje central del discurso legitimador de las diferencias sociales y laborales en el neoliberalismo. En este sentido, la revisión sistemática que presentamos tiene aportaciones teóricas y prácticas que resultan relevantes para la literatura sobre el tema. Como aportaciones teóricas, dota de sistematización a todas las obras que tratan el concepto y que son un marco conceptual para la empleabilidad. Tal y como hemos comentado anteriormente, la gran producción de literatura sobre empleabilidad no se ha construido sobre un constructo con acuerdo científico sobre su significado (Thijssen et al., 2008). De hecho, algunos autores hablan de que el constructo es difuso, poco claro y no es específico. Es más, en muchas ocasiones, no se ha clarificado ni dicho significado ni se ha atendido a la justificación de los indicadores usados. Esto genera imágenes diferenciadas de la empleabilidad y no permiten 
su comparación. Es por ello que diferentes autores de diferentes disciplinas reclaman sistematización de los marcos teóricos y algunos de ellos van más allá y hacen un llamamiento a definiciones y marcos conceptuales integrados (Peeters, Nelissen, De Cuyper, Forrier, Verbruggen y De Whitte, 2019). Junto a ello, dicha dispersión de significados tampoco contribuye a medir a todos los colectivos en igualdad de condiciones. Esto resulta pernicioso, sobre todo, a los grupos vulnerables con dificultad de acceso al empleo. En este sentido, este trabajo aporta a la práctica de inclusión social ya que permite concretar qué se entiende por empleabilidad, cómo se explica y cómo se mide. De esta forma, los agentes sociales vinculados al empleo como por ejemplo los PTA, pueden evaluar, generar y modificar itinerarios formativos para el acceso y el mantenimiento de los puestos de trabajo de los trabajadores de inserción (PTI) en similares condiciones a cualquier otro colectivo (por ejemplo, los jóvenes graduados, las personas que buscan mejorar su puesto de trabajo, etc.). También es una oportunidad para los investigadores de áreas sociales, para los políticos y para todo tipo de profesional vinculados a su evaluación y/o fomento para contribuir a la sostenibilidad de las personas y las sociedades. 


\section{Referencias bibliográficas}

ABDI, A., ESKANDARI, F. \& JALALI, M. (2019): "Comparative analysis of the employability of agricultural and engineering students (the case of Kurdistan University)", Extension and Education Journal, no 15(2), pp. 271-282.

ACENAA, I. \& CAPARRÓS, N. (2018): "Empleabilidad y exclusión social”, Revista Cuadernos de Trabajo Social, no 12(1), pp. 46-63.

AKKERMANS, J., TIMS, M., BEIJER, S. \& DE CUIPER, N. (2019): "Should employers invest in employability? Examining employability as a mediator in HRM-Commitment relations", Frontiers in Psychology, no 10(717), pp. 1-10.

ALTMAN, D.G. (1991): Practical statistics for medical research, London: Chapman $\&$ Hall.

ÁlVAREZ-GONZÁleZ, P., LÓPEZ-MigueNS, M.J. \& CABALlERO, G. (2017): "Perceived employability in university students: developing an integrated model”, Career Development International, no 22(3), pp. 280-299. doi: 10.1108/ CDI-08-2016-0135.

ANDREWS, J. \& HIGSON, H. (2008): "Graduate employability, 'soft skills' versus 'hard' business knowledge: a European study", Higher Education in Europe, no 33(4), pp. 411-422.

ANGUERA, M.T. (1986): "La investigación cualitativa”, Educar, 10, 23-50.

ARESTIS, P., FERREIRO, J. \& GÓMEZ, C. (2020): "Quality of employment and employment protection. Effects of employment protection on temporary and permanent employment", Structural Change and Economic Dynamics, no 53, pp. 180-188. doi: 0.1016/j.strueco.2020.02.008

ARROW, K. (1972): Higher education as a filter. Technological report, USA: University of Stanford.

ARTESS, J., HOOLEY, T. \& MELLORS-BOURNE, R. (2017): Employability: A Review of the Literature 2012-2016, York: Higher Education Academy.

ASHFORD, S.J. \& TAYLOR, M.S. (1990): "Adaptation to work transitions: An integrative approach", Research in Personnel and Human Resources Management, no 8, pp. 1-39.

ATKINS, M.J. (1999): "Oven-ready and self-basting: taking stock of employability skills", Teaching in Higher Education, no 4(2), pp. 267-280. 
La empleabilidad a debate: ¿qué sabemos sobre la empleabilidad como estrategia...

(pp. 313-363)

ATTARWALA, A. \& BALASUBRAMANIAM, C.S. (2019): Enterprise development and employability enhancement: A case study on Corporate Social Responsibility Projects by Kohinoor Technical Institute, Mumbai. Thirteenth Biennial Conference on Entrepreneurship/ Edited by Sasi Misra, Sunil Shukla, Ganapathi Batthini

BAGSHAW, M.(1997): “Employability-Creatinga contract of mutual”, Industrialand Commercial raining, no 29(6), pp. 187-189. doi:10.1108/00197859710177468

BALTANÁS, J. (1999): Empleabilidad: Estrategia compartida, Colección RRHH. Ranstad trabajo temporal.

BANAJI, M. \& GREENWALD, A.G. (1994): "Implicit stereotyping and prejudice". En Ontario symposium on personality and social psychology (Eds. ZANNA, M.P. \& OLSON, J.M.), Vol. 7. The psychology of prejudice: The Ontario symposium, Vol. 7, Lawrence Erlbaum Associates, Inc, pp. 55-76.

BARUCH, Y. (2001): "Employability: A substitute for loyalty?", Human Resource Development International, no 4(4), pp. 543-566.

BATES, L., HAYES, H., WALKER, S. \& MARCHESI, K. (2018): "From employability to employment: A professional skills development course in a three-year bachelor program", International Journal of Work-Integrated Learning, no 19(4), pp. 413-423.

BEDFORD, S. \& BELL, K. (2019): "Curriculum transformation for graduate connectedness and employability: perspectives from the University of Wollongong". En Higher Education and the Future of Graduate Employability (ANGUERA, M.T. (1986): "La investigación cualitativa"), Massachusets, USA: Eduard Elgar Publishing, pp. 160-180. doi: 10.4337/9781788972611

BELT, V. \& RICHARDSON, R. (2005): "Social Labour, Employability and Social Exclusion: Pre-employment Training for Call Centre Work", Urban Studies, n ${ }^{\circ}$ 42(2), pp. 257-270.

BELWAL, R. PRIYADARSHI, P. \& AL FAZARI, M.H. (2016): “Graduate attributes and employability skills. Graduates' perspectives on employers' expectations in Oman", International Journal of Educational Management, no 31(6), pp. 814827. DOI: 10.1108/IJEM-05-2016-0122

BERNAD, J.C., MARTÍNEZ, I. \& MOLPECERES, M.A. (2011): “Trabajo, precariedad y ciudadanía. Las políticas sociolaborales en el contexto del desmantelamiento de la sociedad salarial". En Trabajo, empleabilidad y vulnerabilidad social. (CÓRDOBA, A.I. \& MARTÍNEZ, I.), Universitat de València, 23-59.

BERNSTROM, V.H., DRANGE, I. \& MARMELUND, S.E. (2019): "Employability as an alternative to job security", Personnel Review, no 48 (1), pp. 234-248. doi: 10.1108/PR-09-2017-0279 
BERNTSON, E., SVERKE, M. \& MARKLUND, S. (2006): "Predicting perceived employability: Human capital or labour market opportunities?", Economic and Industrial Democracy, no 27, pp. 223-244.

BHARATHI, A.V. (2016): "Communication skills-core of employability skills: Issues and concerns", Higher Learning Research Communications, no 6(4), pp. 5. Recuperado de https://scholarworks.waldenu.edu/hlrc/vol6/iss $4 / 5 /$ DOI: $10.18870 /$ hlrc.v6i4.358

BLOCH, S. \& BATES, T. (1995): Employability. Your Way to Career Success, London: Kogan Page.

BOLLÉROT, P. (2001): "Two actors of employability: The employer and the worker". En Employability: From theory to practice (Eds. WEINERT, P. , BAUKENS, M., BOLLÉROT, P., PINESCHI-GAPÀNNE, M. \& WALWEI, U.), New Brunswick, London: Transaction Publishers, pp. 3-23.

BONFIGLIOLI, E., MOIR, L. \& AMBROSINI, M. (2006): "Developing the wider role of business in society: The experience of Microsoft in developing training and supporting employability", Corporate Governance, no 6(4), pp. 401- 408. BOURDIEU, P. \& PASSERON, J.C. (1977): La reproducción, Barcelona: Laia.

BRIDGES, W. (1994): Job Shift, Addison-Wesley, Reading, MA.

BRONFENBRENNER, U. \& MORRIS, P. (1998): "The ecology of developmental process". En Handbook of child psychology: Vol.1. Theoretical models of human development (DAMON, W. (Series Ed.) \& LERNER, R.M. (Vol. Ed.)), New York: Wiley, $5^{\text {th }}$ ed., pp. 993-1028.

BRONFENBRENNER, U. \& MORRIS, P. (2006): "The bioecological model of human development". En Handbook of child psychology: Vol.1. Theoretical models of human development (DAMON, W. (Series Ed.) \& LERNER, R.M. (Vol. Ed.)), New York: Wiley, $6^{\text {th }}$ ed., pp. 793-828.

BRONFENBRENNER, U. (1987): La ecología del desarrollo humano. Experimentos en entornos naturales y diseñados, Barcelona: Paidós.

BRONFENBRENNER, U. (Ed.) (2005): Making human beings human: Bioecological perspectives on human development, Thousand Oaks, CA: Sage.

BROWN, P. \& HESKETH, A. (2004): The mismanagement of talent. Employability and jobs in the knowledge economy, New York: Oxford University Press.

BROWN, P., HESKETH, A. \& WILLIAMS, S. (2003): "Employability in a knowledge-driven economy", Journal of Education and Work, no 16(2), 107-126.

DOI: $10.1080 / 1363908032000070648$

BRUNET, I. \& PASTOR, M.I. (2002): "Formación, empleabilidad y desigualdades sociales", Témpora: Revista de Historia y Sociología de la Educación, no 5, pp. 1560. 
La empleabilidad a debate: ¿qué sabemos sobre la empleabilidad como estrategia...

(pp. 313-363)

BUDZYNSKI-SEYMOUR, E., CONWAY, R., WADE, M., LUCAS, A., JONES, M., MANN, S. \& STEELE, J. (2020): "Physical activity, mental and personal well-being, social isolation, and perceptions of academic attainment and employability in University students: The Scottish and British Active Students Survey", Journal of Physical Activity and Health, no 5, pp. 1-11. doi: 10.1123/jpah.20190431.

BUTTERWICK, S. \& BENJAMIN, A. (2006): "The road to employability through personal development: a critical analysis of the silences and ambiguities of the British Columbia (Canada) life skills curriculum", International Journal of Lifelong Education, no 25(1), pp. 75-86. DOI: 10.1080/02601370500309543

CABALlERO, G., VÁZQUEZ, X.H. \& QUINTÁS, M.A. (2015): "Improving employability through stakeholders in European higher education: the case of Spain”, Long Range Planning, no 48(6), pp. 398-411.

CABO, A., PAGÁN, E. \& NIETO, J.G. (2008): "Cuestionario sociométrico de la empleabilidad”, Territorio y Politicas Sociales, no 16. Burjassot: Valencia Consorcio Pactem Nord.

CAMPOS RÍOS, G. (2002): Un modelo de empleabilidad basado en resistencias. El caso del mercado de trabajo en Puebla, Universidad Autónoma Metropolitana. Doctorado en Estudios Sociales. Disponible en http://www.eumed.net

CAMPOS RÍOS, G. (2003): "Implicancias del concepto de empleabilidad en la reforma educativa”, Revista Iberoamericana de educación, Madrid. OEI. Recuperado de www.campos_oei.org/revista/edu_tra2.htlm

CAMPOS, K.C.L., RUEDA, F.J.M., MARTINS, L.J., MANCINI, C.C., GHIRALDELLI, C. \& FUMACHE, R.D. (2003): "Conceito de empregabilidade na visão de alunos de psicologia", Anais do III Congresso Norte-Nordeste de Psicologia, João Pessoa, 422-423.

CARRIERI, A. \& SARSUR, A. (2002): "Percurso semántico do tema empregabilidade: Um estudo de caso em uma empresa de telefonía", Revista de Administração Contemporânea, no 8, pp. 129-150.

CASCIO, W.F. (1993): “Downsizing: what do we know, what have we learned?", Academy of Management Executive, Vol. 7, no 1, pp. 95-104.

CASTEL, R. (2000): Les metamorphoses de la question sociale, Konstanz: Universitätsverlag.

CASTILlO, A. (1999): Estado del arte en la enseñanza del emprendimiento, Programa Emprendedores como creadores de riqueza y desarrollo regional INTEC Chile. <http://www.finam.cl/contenidos/aliciacastilloholley/estado_arte_emprendimiento.pdf>. 
CIESIELKIEWICZ, M. (2019): "Education for employability: the ePortfolio from school principals' perspective", On The Horizon, no 27(1), pp. 46-56.

DOI: 10.1108/OTH-01-2019-0001

CLARKE, M. \& PATRICKSON, M. (2008): "The new covenant of employability”, Employee Relations, no 30(2), pp. 121-141.

CLARKE, M. (2008): "Understanding and managing employability in changing career contexts", Journal of European Industrial Training, no 32(4), pp. 258-284.

CLARKE, M. (2013): "The organizational career: not dead but in need of redefinition”, International Journal of Human Resource Management, n 24(4), pp. 684703.

CLARKE, M. (2018): "Rethinking graduate employability: The role of capital, individual attributes and context", Studies in Higher Education, no 43(11), pp. 19231937.

CONCHADO PEIRÓ, A. \& CAROT SIERRA, J.M. (2013): "Puntos fuertes y débiles en la formación por competencias según los graduados universitarios españoles", REDU. Revista de Docencia Universitaria, no 11(1), pp. 429-446. doi:10.4995/redu.2013.5608

CRANDALL, C.S. (1994): "Prejudice against fat people: Ideology and self-interest", Journal of Personality and Social Psychology, no 66(5), pp. 882-894.

https://doi.org/10.1037/0022-3514.66.5.882

CURRIE, G. (2006): "New careers for old? Organizational and individual responses to changing boundaries", The International Journal of Human Resource Management, no 17(4), pp. 755-774. doi: 10.1080/09585190600581733

DACRE, P.L., QUALTER, P. \& SEWELL, P.J. (2014): "Exploring the factor structure of the career EDG employability development profile", Education+Training, no 56(4), pp. 303-313.

DANIELS, J., D’ANDREA, M. \& GAUGHEN, K.J.S. (1998): “Testing the validity and reliability of the Perceived Employability Scale (PES) among a culturally diverse population", Journal of Employment Counseling, no 35(3), pp. 114-123.

DAVIS, C., BATY, B.J., HIPPMAN, C., TREPANIER, A. \& ERBY, L. (2019): "Genetic counselors with advanced skills: II. A new career trajectory framework", Journal of Genetic Counseling, no 0, pp. 1-15. doi. 10.1002/jgc4.1204

DE LARA, K.C. \& ANDRADE, F. (2008): "Empregabilidade: Construçao de uma escala”, Psico-USF, no 13(2), pp. 189-201.

DUCCI, M.A. (1998): "La formación al servicio de la empleabilidad", Boletín Cinterfor: Boletín Técnico Interamericano de Formación Profesional, no 142, pp. 7-23. 
La empleabilidad a debate: ¿qué sabemos sobre la empleabilidad como estrategia...

(pp. 313-363)

EBUENYI, I.D., GUXENS, M., OMBATI, E., BUNDERS-AELEN, J.F.G. \& REGEER, B.J. (2019): "Employability of perons with mental disability: Understanding lived experiences in Kenya", Frontiers in Psychiatry, no 10(539), pp. $1-13$.

ELLIG, B.R. (1998): "Employment and employability: Foundation of the new social contract", Human Resource Management, no 37(2), pp. 173-175.

ENRÍQUEZ, A. \& RENTERÍA, E. (2007): "Estrategias de aprendizaje para la empleabilidad en el mercado de trabajo de profesionales recién egresados", Universitas Psychologica, no 6, pp. 89-103.

ESPINOZA, O., GONZÁLEZ, L.E., MCGINN, N., SANDOVAL, L. \& CASTILLO, D. (2020): "Should universities train teachers for employability or for effectiveness?", Teaching and Teacher Education, no 88, pp. 102960.

FAHIMIRAD, M., NAIR, P.K., KOTAMJANI, S.S., MAHDINEZHAD, M. \& FENGF, J.B. (2019): "Integration and development of employability skills into Malaysian Higher Education context: Review of the literature", International Journal of Higher Education, no 8(6), pp. 26-35.

FAULKNER, P.E., BEGUM, S. \& ENGLISH, C.W. (2019): "How Bangladesh Is Increasing the Employability of Females With Technical Training Programs". In Gender Issues in Technical and Vocational Education Programs (Ed. BALA, S. \& SINGHAL, P.), IGI Global, pp. 158-182.

DOI:10.4018/978-1-5225-8443-8.ch008

FERNS, S., DAWSON, V. \& HOWITT, C. (2019): "A collaborative framework for enhancing graduate employability", International Journal of Work-Integrated Learning, no 20(2), pp. 99-111.

FERREIRA, I., URRÚTIA, G. \& ALONSO-COELLO, P. (2011): "Revisiones sistemáticas y metaanálisis: bases conceptuales e interpretación”, Revista Española de Cardiología, no 64(8), pp. 688-696.

FINCH, D.J., PEACOCK, M., LEVALLET, N. \& FOSTER, W. (2015): “A dynamic capabilities view of employability. Exploring the drivers of competitive advantage for university graduates", Education + Training, no 58(1), pp. 61-81. DOI: $10.1108 /$ ET-02-2015-0013

FINN, D. (2000): "From full employment to employability: a new deal for Britain's unemployed?", International Journal of Manpower, no 21(5), pp. 384-399.

FORMICHELLA, M.M. \& LONDON, S. (2005): Reflexiones acerca de la noción de empleabilidad. Anales, Trabajo presentado en las reuniones de la AAEP. Buenos Aires Argentina: Recuperado de http://www.aaep.org.ar/espa/anales/works05/ formichella_london.pdf 
FORRIER, A. \& SELS, L. (2003): "The concept of employability: a complex mosaic", International Journal of Human Resources Development and Management, $\mathrm{n}^{\circ}$ 3(2), pp. 102-124.

FOX, D.J. (1981): El proceso de investigación en educación, Pamplona: EUNSA.

FUGATE, M. \& KINICKI, A.J. (2008): "A dispositional approach to employability: Development of a measure and test of implications for employee reactions to organizational change", Journal of Occupational and Organizational Psychology, $\mathrm{n}^{\circ}$ 81(3), pp. 503-527.

FUGATE, M., KINICKI, A.J. \& ASHFORTH, B.E. (2004): "Employability: a psycho-social construct, its dimensions, and applications", Journal of Vocational Behavior, no 65(1), pp. 14-38.

FUSTER, J. (1999): Empleabilidad: la capacidad de una persona. Disponible en: http://www.fundipe.es.

GAMBOA, J.P., GRACIA, F.J., RIPOLL, P. \& PEIRÓ, J.M. (2007): La empleabilidad y la iniciativa personal como antecedentes de la satisfacción laboral. Disponible en: https://web2011.ivie.es/downloads/docs/wpasec/wpasec-2007-01.pdf

GARZSO, A. (2007): "Balancing expectations for employability and family responsibilities while on social assistance: Low-income mothers' experiences in three Canadian provinces”, Family Relations, no 56(5), pp. 454-466.

GAZIER, B. (1998): "Observations and recommendations". En Employability-Concepts and policies (Ed. GAZIER, B.), Berlin: European Employment Observatory, pp. 298-315.

GAZIER, B. (2001): "Employability: The complexity of a policy notion". En Employability: From theory to practice (Eds. WEINERT, P., BAUKENS, M., BOLLÉROT, P., PINESCHI-GAPÀNNE, M. \& WALWEI, U.), New Brunswick, London: Transaction Publishers, pp. 3-23.

GÓMEZ-TORRES, M.J., RODRÍGUEZ-SANTERO, J. \& GIL-FLORES, J. (2019): "Job-search strategies of individuals at risk of poverty and social exclusion in Spain", Plos One, no 14(1), pp. 1-13. DOI: 10.1371/journal.pone.0210605

GORDON, D.M., EDWARS, R. \& REICH, M. (1982): Segmented work, divided workers: The historical transformation of labor in the United States, New York: Cambridge University Press.

GRIP, A., VAN LOO, J. \& SANDERS, J. (1999): Employability in action: An industry employability index. Skope Research paper, no 5 . Recuperado de http://ora. ox.ac.uk/objects/uuid\%3Ac546634d-f562-4971-ad56-0115e5065f00/datastreams/ATTACHMENT01

HALL, D.T. (2002): Careers in and out of organizations, Thousand Oaks: Sage. 
La empleabilidad a debate: ¿qué sabemos sobre la empleabilidad como estrategia...

(pp. 313-363)

HARVEY, L. (2001): "Defining and measuring employability", Quality in Higher Education, no 7(2), pp. 97-109.

HAZELZET, E., PICCO, E., HOUKES, I., BOSMA, H. \& DE RIJK, A. (2019): "Effectiveness of interventions to promote sustainable employability: A systematic review", International Journal of Environmental Rearch and Public Health, $\mathrm{n}^{\circ}$ 16, doi:10.3390/ijerph16111985

HILLAGE, J. \& POLLARD, E. (1998): "Employability: Developing a framework for policy analysis", EfEE Research Brie.ng, no 85. Institute for Employment Studies. Disponible en http://www.dfes.gov.uk/research/programmeofresearch/ projectinformation.cfm? projectid $=12855$ yresultspage $=1$.

HIND, P. (2005): "Making room for career change", Career Development International, no 10(4), pp. 268-74.

HIRATA, H. (1997): Os mundos do trabalho. En Empregabilidade e Educação. Novos caminhos no mundo do trabalho (Orgs. CASALI, A. RIOS, I., TEIXEIRA, J.E. \& CORTELLA, Y.M.S.), São Paulo: EDUC- RHODIA, pp. 23-42.

INKSON, K., GUNZ, H., GANESH, S. \& ROPER, J. (2012): "Boundaryless careers: bringing back boundaries”, Organization Studies, no 33(3), pp. 323-340. doi: 10.1177/0170840611435600

JOST, J.T. \& BANAJI, M.R. (1994): “The role of stereotyping in system-justification and the production of false consciousness", British Journal of Social Psychology, no 33(1), pp. 1-27. doi: 10.1111/j.2044-8309.1994.tb01008.x

KANFER, R., WANBERG, C.R. \& KANTROWITZ, T.M. (2001): "Job search and employment: A personality-motivational analysis and meta-analytic review", Journal of Applied Psychology, no 86, pp. 837-855.

KEELEY, B. (2007): Capital humano: Cómo influye en su vida lo que usted sabe, París: OCDE.

KENDALL, A. \& FRENCH, A. (2018): "Re-thinking employability with a literacies lens. From skills to practices, from tool-kits to ethnography", Higher Education, Skills and Work-Based Learning, no 8(2), pp. 164-178.

doi: 10.1108/HESWBL-09-2017-0055

KENNEDY, M.M. (2007): “Defining a literature”, Educational Researcher, no 36(3), pp. 139-147.

KITTISAK, J., THANAPORN, S. \& PARINYA, S. (2019): “Empirical framework to understand the antecedents of work engagement and employability in the Thai Universities", International Journal of Psychosocial Rehabilitation, $\mathrm{n}^{\circ}$ 23(4), pp. 837-852. 
KOE, WL. (2019): "Individual Entrepreneurial Orientation and Perceived Employability Among University Graduates". En Proceedings of the Regional Conference on Science, Technology and Social Sciences (Eds. MOHAMAD NOOR, M., AHMAD, B., ISMAIL, M., HASHIM H. \& ABDULLAH BAHARUM, M.), Springer, Singapore, (RCSTSS 2016).

KOLOBA, H.A. (2017): "Perceived employability of university students in South Africa. Is it related to employability skills?", International Journal of Social Sciences and Humanity Studies, no 9(1), pp. 73-90.

KOVALENKO, M. \& MORTELMANS, D. (2016): "Contextualizing employability. Do boundaries of self-directedness vary in different labor market groups?", Career Development International, no 21(5), pp. 498-517.

DOI: $10.1108 / C D I-01-2016-0012$

KRIPPENDORFF, K. (1990): Metodología del análisis de contenido. Teoría y práctica, Barcelona: Paidós.

KRIPPENDORFF, K. (2013): Content analysis: an introduction to its methodology, Los Angeles / London: Sage.

LEGGE, K. (2005): Human Resource Management: Rhetorics and realities, New York: Palgrave Macmillan.

LISBONA, A., PALACÍ, F.J. \& MORIANO, J.A. (2002): "La empleabilidad como estrategia de integración y desarrollo". En La persona en la empresa. Iniciativas de integración y desarrollo (PALACÍ, F.J. \& TOPA, G.), Madrid: UNED, pp. 137150.

LLINARES, L.I., CÓRDOBA, A.I. \& ZACARÉS, J.J. (2011): "La medida de la empleabilidad desde las Empresas de Inserción Social”. En Trabajo, empleabilidad y vulnerabilidad social: condicionantes y potencialidades de la integración a través de las Empresas de Inserción Social (CÓRDOBA, A.I. \& MARTÍNEZ, I.), Valencia, SPUV.

LLINARES INSA, L.I., ZACARÉS GONZÁLEZ, J.J. \& CÓRDOBA IÑESTA, A.I. (2016): "Discussing employability: current perspectives and key elements form a bioecological model", Employee Relations, no 38(6), pp. 961-974.

LLINARES INSA, L.I., GONZÁLEZ NAVARRO, P., ZACARÉS, J.J. \& CÓRDOBA IÑESTA, A.I. (2018): "Employability Appraisal Scale (EAS): Development and Validation in a Spanish Sample", Frontiers In Psychology. Section Organizational Psychology, 9, no 1437, pp. 1-11.

DOI: https://doi.org/10.3389/fpsyg.2018.01437 
La empleabilidad a debate: ¿qué sabemos sobre la empleabilidad como estrategia...

(pp. 313-363)

LUGO, M. \& ALCÁNTARA, L. (2017): “Herramientas en la autogestión sostenible para la empleabilidad", Congreso Internacional de Pedagogía Social. Pedagogía social y Desarrollo Humano. XXX Seminario Interuniversitario de Pedagogía Social, 8-10 de noviembre de 2017.

MÄKIKANGAS, A., DE CUYPER, N., MAUNO, S. \& KINNUNEN, U. (2013): "A longitudinal person-centred vie won perceived employability: The role of job insecurity", European Journal of Work and Organizational Psychology, no 22(4), pp. 490-503. DOI: 10.1080/1359432X.2012.665230

MARTÍN, M. \& SPINOZA, A.A. (2005): "Del empleo a la empleabilidad, de la educación a la educabilidad. Mutaciones conceptuales e individualización de los conflictos sociales", Séptimo Congreso de Especialista en Estudios del Trabajo, Buenos Aires, 10-12 de agosto.

MARTÍNEZ CERDÁ, J.F. \& TORRENT SELLENS, J. (2017): "Formal lifelong E-learning for employabiliy and job stability during turbulent times in Spain", International Review of Research in Open and Distributed Learning, no 18(6), pp. 261-287.

MARTÍNEZ CERDÁ, J.F., TORRENT SELLENS, J., GONZÁLEZ GONZÁLEZ, I. \& FICAPAL CUSÍ, P. (2018): "Opening the black-box in lifelong e-learning for employability: A framework for a socio-tecnical e-learning employability system of measurement (STELEM)", Sustainability, no 10(1014), pp. 1-27.

DOI:10.3390/su10041014

MCARDLE, S., WATERS, L., BRISCOE, J.P. \& HALL, D.T.T. (2007): “Employability during unemployment: adaptability, career identity and human and social capital”, Journal of Vocational Behavior, no 71(2), pp. 247-264.

MCQUAID, R., GREEN, A. \& DANSON, M. (2005): "Introducing employability", Urban Studies, no 42(2), pp. 191-195.

MCQUAID, R.W. \& LINDSAY, C. (2005): "The concept of employability", Urban Studies, no 42(2), pp. 197-219.

MIDTSUNDSTAD, T. (2019): "A review of the research literature on adult learning and employability", European Journal of Education, no 54, pp. 13-29.

DOI: 10.1111/ejed.12321

MOHAMAD, M., JAMALUDIN, H., ZAWAEI, A.A. \& WAN HANAFI, W.N. (2018): "Determinants influencing employability skills: Undergraduate perception", Global Business and Management Research: An International Journal, $\mathrm{n}^{\circ}$ 10(3), pp. 568-578.

MONTANAANA, J. (2005): Educar las competencias como herramienta de empleabilidad. Consultado el 14 de octubre de 2011 en: http://www.educaweb.com/educa. news $/$ interface $/$ asp $/$ web/noticiesmostrar.asp? noticiaid $=341$ yseccioid $=539$ 
MORELAND, N. (2006): Entrepreneurship and higher education: An employability perspective, Heslington, York: Enhancing Student Employability Co-ordination Team, ESECT.

MORENO, M.P., SANTOS, J.A., RAMOS, L.A., SANZ, D., FUENTES, J.P. \& DEL VILLAR, F. (2002): "Aplicación de un sistema de codificación para el análisis de contenido de la conducta verbal del entrenador de voleibol", Revista Motricidad, 9, 119-140.

MORRISON, E.W. \& ROBINSON, S.L. (1997): "When employees feel betrayed: a model of how psychological contract violation develops", The Academy of Management Review, no 22(1), pp. 226-256.

MUNIZ, L. (2012): “Carreras y trayectorias laborales: Una revisión crítica de las principales aproximaciones teórico-metodológicas para su abordaje”, Revista Latinoamericana de Metodología de las Ciencias Sociales, no 2(1), pp. 36-65.

NALAWADE, R.K., MORE, D.K. \& BHOLA, S.S. (2019): "Employability skills required for functional areas of management", The IUP Journal of Soft Skills, no XIII(1), pp. 20-44.

NAUTA, A., VAN VIANEN, A., VAN DEL HEIJDEN, B., VAN DAM, K. \& WILLEMSEN, M. (2009): "Understanding the factors that promote employability orientation: The impact of employability culture, career satisfaction, and role breadth self-efficacy", Journal of Occupational and Organizational Psychology, no 82, pp. 233-251. DOI: 10.1348/096317908X320147

NISHA, S. \& RAJASEKARAN, V. (2018): "Employability skills: A review", The IUP Journal of Soft Skills, no XII(1), pp. 29-37.

OLSSEN, M. \& PETERS, M.A. (2005): "Neoliberalism, higher education and the knowledge economy: from the free market to knowledge capitalism", Journal of Education Policy, 20(3), 313-345. DOI: 10.1080/0268093050018718

ORELLANA, N. (2018): "Consideraciones sobre empleabilidad en educación superior", Calidad en la Educación, no 48, pp. 273-291.

PEETERS, E., NELISSEN, J., DE CUYPER, N., FORRIER, A., VERBRUGGEN, M. \& DE WITTE, H. (2019): "Employability Capital: A Conceptual Framework Tested Through Expert Analysis", Journal of Career Development, no 46(2), pp. 79-93.

PEIRÓ, J.M. \& YEVES, J. (2017): Análisis de la empleabilidad, sus antecedentes y Consecuencias, Atti del convegno "Work in progress" for a better quality of life. DOI: $10.1285 / 9788883051289 \mathrm{p} 23$

PÉREZ, F. (Dir.) (2018): Itinerarios de inserción laboral y factores determinantes de la empleabilidad. Formación universitaria vs entorno, Bilbao: Fundación BBVA. 
La empleabilidad a debate: ¿qué sabemos sobre la empleabilidad como estrategia...

(pp. 313-363)

PÉREZ, P. (2005): "Sobreeducación, desclasamiento y la compleja relación entre educación y empleo". En Anales del V Encuentro Internacional de Economía, Buenos Aires: Editorial CIEC, 63-65.

PETER, J. \& GÓMEZ, S.J. (2019): "Skill building for employability", The IUP Journal of Soft Skills, no XII(3), pp. 42-55.

PHILIPPAERS, K., DE CUIPER, N. \& FORRIER, A. (2019): "Employability and performance. The role of perceived control and affective organizational commitment”, Personnel Review, no 48(5), pp. 1299-1317.

DOI: $10.1108 /$ PR-04-2017-0098

PIORE, M.J. (1995): Beyond Individualism, Cambridge, Harvard University Press.

PITAN O.S. \& MULLER, C. (2019): “University reputation and undergraduates' self-perceived employability: mediating influence of experiential learning activities", Higher Education Research \& Development, no 38(6), pp. 1269-1284.

DOI: 10.1080/07294360.2019.1634678

PRIETO, C. (1999): “Crisis del empleo, ¿crisis del orden social?” En Las relaciones de empleo en España (Eds. MIGUÉLEZ, F. \& PRIETO, C.), Madrid: Siglo XXI.

RAE, D. (2007): "Connecting enterprise and graduate employability", Education+Training, no 49(8/9), pp. 605-619.

RAJAN, A. (2000): Employability: Bringing the gap between rhetoric and reality: First Report: Employers' perspective, Centre for Research in Employment \& Tech in Europe.

RAJAN, A., VAN EUPEN, P., CHAPPLE, K. \& LANE, D. (2000): Employability: Bridging the Gap between Rhetoric and Reality, First Report: Employer's Perspective, Create Consultancy/Professional Development Foundation, London.

RAMOS, J. (Dir.) (2010): El empleo y la dimensión social en la estrategia UE-2020, Madrid: Ministerio de Trabajo e Inmigración. Subdirección General de Publicaciones.

RÄTY, H., KOMULAINEN, K., HARVORSÉN, C., NIEMINEN, A. \& KORHONEN, M. (2018): "University students' perceptions of their 'ability selves' and employability: a pilot study", Nordic Journal of Studies in Educational Policy, no 4(2), pp. 107-115. DOI: 10.1080/20020317.2018.1453221

REID, J. (2015): "Redefining "Employability" as something to be achieved. Utilizing Tronto's conceptual framework of care to refocus the debate", Higher Education, Skills and Work-Based Learning, no 6(1), pp. 55-68.

doi: 10.1108/HESWBL-02-2015-0005

RENTERÍA, E. (2005): "Empleabilidad: Una lectura psicosocial", Revista de Estudios del Trabajo en Colombia, no 1(1), pp. 1-14. 
RENTERÍA-PÉREZ, E. \& MALVEZZI, S. (2008): "Empleabilidad, cambios y exigencias psicosociales en el trabajo", Universitas Psychologica, no 2(2), pp. 319-334. RIQUELME, G.R. \& RAZQUIN, P. (1999): "Mercado de trabajo y educación: el papel de la educación en el acceso al empleo", Revista de Ciencias Sociales, no 12(16), pp. 1-24.

RÖMGENS, I., SCOUPE, R. \& BEAUSAERT, S. (2019): "Unraveling the concept of employability, bringing together research on employability in higher education and the workplace", Studies in Higher Education.

DOI: $10.1080 / 03075079.2019 .1623770$

ROTHWELL, A. \& ARNOLD, J. (2007): "Self-perceived employability: Development and validation of a scale", Personnel Review, no 36(1), pp. 23-41.

ROTHWELL, A., HERBERT, I. \& ROTHWELL, F. (2008): "Self-perceived employability: construction and initial validation of a scale for university students", Journal of Vocational Behavior, no 73(1), pp. 1-12.

ROUSSEAU, D.M. (1995): Psychological Contracts in Organizations: Understanding Written and Unwritten Agreements, Sage Publications, Thousand Oaks, CA.

ROZALÍA, M., BLAGA, P. \& MATIS, C. (2019): "Supporting employability by a skills assessment innovative tool-sustainable transnational insights from employers", Sustainability, no 11(12), pp. 3360. Doi: 10.3390/su11123360

SABIN, C. \& HANSVICK, C.L. (1999): "Applicant age as a subjective employability factor: A study workers over and under age fifty2", Journal of Employment Counseling, no 36(1), pp. 24-34.

SALANOVA, M. \& LLORENS, S. (2006): "Desarrollando la empleabilidad de los Recursos Humanos: un reto de nuestro tiempo", Estudios Financieros, no 227, pp. 225-240.

SALOGNON, M. (2007): "Reorienting companies' hiring behaviour: an innovative 'back-to-work' method in France", Work, employment y society, no 21, pp. 713730. doi: 0.1177/0950017007082878

SANTANA, L.E., ALONSO, E. \& FELICIANO, L. (2016): "Trayectorias laborales y competencias de empleabilidad de jóvenes nacionales e inmigrantes en riesgo de exclusión social”, Revista Complutense de Educación, no 29(2), pp. 355-369.

SARMENTO, M., FERREIRA, M., AMORIM, M., MADALENO, M. \& PIMENTEL, C. (2019): "Transversal competences towards employability in female students". En Handbook of Research on Women in Management and the Global Labor Market (Eds. PEREIRA, E.T. \& PAOLONI, P.), Pennsylvania, USA: IGi Global, pp. 63-90.

SCHEIBELHOFER, E. \& TÄUBIG, V. (2019): "Beyond employability: refugees working lives”, Identities, no 26(3), pp. 261-269.

DOI: 10.1080/1070289X.2019.1590027 
La empleabilidad a debate: ¿qué sabemos sobre la empleabilidad como estrategia...

(pp. 313-363)

SENNET, R. (2010): L'uomo flessiblile. Le consequenze del nuovo capitalismo sulla vita delle persone, Milano: Feltrinelli.

SERRANO, M.A. (2000): "El concepto de empleabilidad en la estrategia europea de lucha contra el desempleo: Una perspectiva crítica", Revista de Ministerio de Trabajo y Asuntos Sociales, no 21, pp. 137-150. Madrid.

SHARMA, G.V. \& SHIVAKUMAR, J. (2019): "Project Assignment as an Effective Method to Enhance the Communication Skills of Business Management Students", IUP Journal of English Studies, no 14 (3), pp. 73-80.

SINGH, R., CHAWLA, G., AGARWAL, S. \& DESAI, A. (2016): "Employability and innovation: Development of a scale", International Journal o Innovation Science, $\mathrm{n}^{\circ}$ 9(1), pp. 20-37. doi: 10.1108/IJIS-10-2016-0041

SISODIA, S. \& AGRAWAL, N. (2019): "Examining employability skills for healthcare services in India: A descriptive literature review", International Journal of Service Science, Management, Engineering, and Technology, no 10(3), pp. 63-79. doi: 10.4018/IJSSMET.2019070105

SJOLLEMA, S. (2007): "Naar een nuieuw psychologisch contract" (Towards a new pshcological contract), NScP Journaal, no 3(3), pp. 6-7.

SMITH, J., MCKNIGH, A. \& NAYLOR, R. (2000): "Graduate employability: Policy and performance in higher education in the UK", The Economic Journal, no 110, pp. 382-411. DOI: 10.1111/1468-0297.00546

STORME, E., DE LANNOY, A., LEIBBRANDT, M., DE BOEXK, F. \& MUDIRIZA, G. (2019): Developing a multidimensional youth employability. Index to unpack vulnerabilities in the lived realities of youth in Post-apartheid South Africa, Cape Town: SALDRU, UCT (Working Paper Series, 255).

SUÁREZ, B. (2016): "Empleabilidad: análisis del concepto", Revista de Investigación en Educación, no 14(1), pp. 67-84.

TEJAN, O.A. \& SABIL, A. (2019): "Understanding employers' perception of employability skills and career development in Morocco", International Journal of Education y Literacy Studies, no 7(2), pp. 134-138.

doi: $10.7575 /$ aiac.ijels.v.7n.2p.134

TERZALORY, C. \& OYEKUNLE, Y. (2019): "Career service as a measure to support employability: A comparison between the univsersity of Florence and the University of Lagos", Andragoška spoznanja/Studies in Adult Education and Learning, $\mathrm{n}^{\circ}$ 25(1), pp. 89-110. doi. 10.4312/as.25.1.89-110

TEZANOS, J.F. (2001): La sociedad dividida. Estructuras de clases y desigualdades en las sociedades tecnológicas, Madrid: Biblioteca Nueva.

THIEME, D. (2007): "El desarrollo de competencias de empleabilidad en dos universidades chilenas: un estudio empírico", Revista OIKOS, no 11(24), pp. 47-72. 
THIJSSEN, J.G.L. \& VAN DER HEIJDEN, B.I.J.M. (2003): "Employability in the focus of attention. Onset to enlightening a confuse phenomenon", Paper presented at the 7th Conference on International Human Resource Management.

THIJSSEN, J.G.L., VAN DER HEIJDEN, B.I.J.M. \& ROCCO, T.S. (2008): "Toward the Employability-Link Model: Current Employment Transition to Future Employment Perspectives", Human Resource Development Review, no 7(2), pp. $165-183$.

THUROW, L. (1972): "Education and Economic Inequality", The Public Interest, no 28, pp. 66-81.

TOMLINSON, M. (2012): "Graduate employability: A review of conceptual and empirical themes”, Higher Education Policy, no 25, pp. 407-431.

DOI:10.1057/hep.2011.26

TORRES, O. (2000): Empregabilidade negociada, São Paulo: Atlas.

URRÚTIA, G. \& BONFILL, X. (2010): "Declaración PRISMA: Una propuesta para mejorar la publicación de revisiones sistemáticas y metaanálisis", Medicina Clínica, no 11(9), pp. 507-511.

VALERO, I. (2019): "La transformación del trabajo en España desde los años setenta hasta hoy: una mirada generacional", Studia Policite, no 47, pp. 45-71.

VAN BUREN, H.J. (2003): "Boundaryless careers and employability obligations", Business Ethics Quarterly, no 13(2), pp. 131-149.

VAN DE HEIJDEN, C.M. \& VAN DER HEIJDEN, B.I.J.M. (2005): "The development and psychometric evaluation of a multi-dimensional measurement instrument of employability-and the impact of aging", International Congress Series, no 1280 , pp. 142-147. DOI: 10.1016/j.ics.2005.02.061

VAN DER HEIJDE, C.M. \& VAN DER HEIJDEN, B.I.J.M. (2006): "A Competence-based and Multidimensional Operationalization and Measurement of Employability”, Human Resource Management, no 45(3), pp. 449-476.

VAN DER HEIJDEN, B. \& SPURK, D. (2019): "Moderating role of LMX and proactive coping in the relationship between learning value of the job and employability enhancement among academic staff employees", Career Development International, no 24(2), pp. 163-186. doi: 10.1108/CDI-09-2018-0246

VARGAS, R., SÁNCHEZ-QUEIJA, M.I., ROTHWELL, A. \& PARRA, A. (2018): "Self-perceived employability in Spain", Education + Training, no 60(3), pp. 226-237. DOI: 10.1108/ET-03-2017-0037

WEINBERG, P. (2004): Formación profesional, empleo y empleabilidad. Disponible en: http://www.cinterfor.org.uy/public/spanish/region/ampro/cinterfor/publ/ sala/ weinberg/index.htm. 
WILLIAMS, S., DODD, L.J., STEELE, C. \& RANDALL, R. (2016): “A systematic review of current understandings of employability", Journal of Education and Work, no 29(8), pp. 877-901. doi: 10.1080/13639080.2015.1102210

WU, Y.L. (2019): "Examining self-perceived employability among immigrant women participating in vocational training in Taiwan", International Journal of Training and Development, no 23(4), pp. 313-327. doi: 10.1111/ijtd.12164

YEVES, J., BRAGSTEDE, M., CORTES, L., MERINO, C. \& CAVADA, G. (2019): "Age and perceived employability as mderators of job insecurity and job satisfaction: A moderated moderation model”, Frontiers in Psychlogy, no 10(799), pp. 1-12.

YIZHONG, X., LIN, Z., BARANCHENKO, Y., LAU, C.K., YUKHANAEV, A. \& LU, H. (2017): "Employability and job search behavior", Employability Relations, $\mathrm{n}^{\circ}$ 39(2), pp. 223-239. DOI: 10.1108/ER-02-2016-0042

YORKE, M. (2006): Employability in higher education: What it is-what it is not, York: Higher Education Academy.

ZHIWEN, G. \& VAN DER HEIJDEN, B. (2008): "Employability enhancement of business graduates in China”, Education+Training, no 50(4), pp. 289-304. 